\title{
APPROXIMATION OF THERMAL EQUILIBRIUM FOR QUANTUM GASES WITH DISCONTINUOUS POTENTIALS AND APPLICATION TO SEMICONDUCTOR DEVICES*
}

\author{
CARL L. GARDNER ${ }^{\dagger}$ AND CHRISTIAN RINGHOFER ${ }^{\dagger}$
}

\begin{abstract}
We derive an approximate solution valid to all orders of $\hbar$ to the Bloch equation for quantum mechanical thermal equilibrium distribution functions via asymptotic analysis for high temperatures and small external potentials. This approximation can be used as initial data for transient solutions of the quantum Liouville equation, to derive quantum mechanical correction terms to the classical hydrodynamic model, or to construct an effective partition function in statistical mechanics. The validity of the asymptotic solution is investigated analytically and numerically and compared with Wigner's $O\left(\hbar^{2}\right)$ solution. Since the asymptotic analysis results in replacing second derivatives of the potential in the correction to the stress tensor in the original $O\left(\hbar^{2}\right)$ quantum hydrodynamic model by second derivatives of a smoothed potential, this approach represents a definite improvement for the technologically important case of piecewise continuous potentials in quantum semiconductor devices.
\end{abstract}

Key words. quantum gases, quantum hydrodynamics, nonlinear PDEs, conservation laws, semiconductor device simulation

AMS subject classifications. 76M20, 76W05, 76Y05

PII. S0036139996303907

1. Introduction. Semiconductor devices that rely on quantum tunneling through potential barriers are playing an increasingly important role in advanced microelectronic applications, including multiple-state logic and memory devices and high frequency oscillators. The propagation of electrons and holes in the semiconductor device can be modeled as the flow of a continuous charged quantum gas in a potential that has discontinuous jumps at heterojunction barriers. The fluid dynamical equations are derived by assuming the gas is near thermal equilibrium, but are expected to be more generally valid.

This investigation is therefore concerned with the approximate description of thermal equilibrium quantum mechanical systems of particles in a potential - especially in the presence of discontinuous potential barriers. Under the assumption of Boltzmann statistics, thermal equilibrium of a quantum mechanical system is described by the factor $\exp \left\{-\beta^{*}(H(V)+\phi)\right\}$, where $H(V)$ is the Hamiltonian operator, $V$ is the potential energy, $\beta^{*}$ is the reciprocal value of the ambient temperature, and $\phi$ is the Fermi level. Depending on the choice of representation of the system, the term $\exp \left\{-\beta^{*}(H(V)+\phi)\right\}$ can be expressed in various ways. Choosing a representation via Schrödinger wavefunctions amounts to solving the eigenvalue problem

$$
E_{\lambda} \psi_{\lambda}(x)=H(V) \psi_{\lambda}(x), \quad H(V)=-\frac{\hbar^{2}}{2 m} \Delta_{x}+V(x), \quad x \in R^{d},
$$

where $d=1,2$, or $3, m$ is the particle mass, and the $\psi_{\lambda}(\lambda=0,1,2, \ldots)$ are the particle wavefunctions. Observable quantities like the thermal equilibrium (denoted

\footnotetext{
* Received by the editors May 20, 1996; accepted for publication (in revised form) December 13, 1996; published electronically March 24, 1998.

http://www.siam.org/journals/siap/58-3/30390.html

${ }^{\dagger}$ Department of Mathematics, Arizona State University, Tempe, AZ 85287-1804 (gardner@ asu.edu, ringhofer@asu.edu). The research of the first author was supported in part by U.S. Army Research Office grant DAAH04-95-1-0122, and the research of the second author was supported in part by ARPA grant F49620-93-1-0062.
} 
by the subscript $e$ ) particle density $n_{e}(x)$, momentum density $\pi_{e}(x)$, and momentum flux density tensor $\Pi_{e}(x)$ are computed as

$$
\begin{gathered}
n_{e}(x)=\sum_{\lambda} \exp \left\{-\beta^{*}\left(E_{\lambda}+\phi\right)\right\}\left|\psi_{\lambda}(x)\right|^{2}, \\
\pi_{e}(x)=\frac{\hbar}{i} \sum_{\lambda} \exp \left\{-\beta^{*}\left(E_{\lambda}+\phi\right)\right\} \psi_{\lambda}^{*}(x) \nabla_{x} \psi_{\lambda}(x), \\
\Pi_{e}(x)=\frac{\hbar^{2}}{m} \sum_{\lambda} \exp \left\{-\beta^{*}\left(E_{\lambda}+\phi\right)\right\}\left\{\nabla_{x} \psi_{\lambda}^{*}(x)\right\} \nabla_{x} \psi_{\lambda}(x) .
\end{gathered}
$$

The summation over the index $\lambda$ is replaced by an integral where the spectrum of the Hamiltonian is continuous.

Fluid dynamical equations are usually formulated in terms of the stress tensor $P_{j k}$, which is related to the momentum flux density tensor by

$$
\Pi_{j k}=\frac{1}{m n} \pi_{j} \pi_{k}-P_{j k},
$$

where $j, k=1, \ldots, d$. The energy density $W$ is calculated from the momentum flux density as

$$
W=\frac{1}{2} \Pi_{j j} .
$$

We will use the summation convention where repeated Latin indices $j, k$ are summed over.

For many applications it is advantageous to express the term $\exp \left\{-\beta^{*}(H(V)+\phi)\right\}$ via the density matrix

$$
\rho_{e}(x, y, \beta)=\sum_{\lambda} \exp \left\{-\beta\left(E_{\lambda}+\phi\right)\right\} \psi_{\lambda}(x) \psi_{\lambda}^{*}(y) .
$$

It can be easily shown [12] that if the wavefunctions form an orthonormal eigensystem, the density matrix satisfies the initial value problem for the Bloch equation

$$
\begin{gathered}
\partial_{\beta} \rho_{e}=\frac{\hbar^{2}}{4 m}\left(\Delta_{x}+\Delta_{y}\right) \rho_{e}-\frac{1}{2}[V(x)+V(y)] \rho_{e}-\phi \rho_{e}, \\
\rho_{e}(x, y, \beta=0)=\delta(x-y) .
\end{gathered}
$$

Thus, solving the eigenvalue problem for the Schrödinger equation is replaced by solving a parabolic initial value problem in twice as many dimensions. The Bloch equation is the interpretation of the term $\exp \left\{-\beta^{*}(H+\phi)\right\}$ as a semigroup.

The initial condition (9) means that we prescribe randomized behavior at infinite temperature $(\beta=0)$ and solve the Bloch equation up to the specified ambient temperature $T_{0}=1 / \beta^{*}$. (We set Boltzmann's constant $k_{B}=1$.) In the density matrix representation, the particle, momentum, and momentum flux densities are

$$
n_{e}(x)=\rho_{e}\left(x, x, \beta^{*}\right),
$$




$$
\begin{gathered}
\pi_{e}(x)=\left.\frac{\hbar}{i} \nabla_{x} \rho_{e}\left(x, y, \beta^{*}\right)\right|_{y=x}, \\
\Pi_{e}(x)=\left.\frac{\hbar^{2}}{m} \nabla_{x} \nabla_{y} \rho_{e}\left(x, y, \beta^{*}\right)\right|_{y=x} .
\end{gathered}
$$

To relate the quantum mechanical description to the classical picture, we use the Wigner transform of the Bloch equation. The Wigner (distribution) function [14] is

$$
w(x, p, \beta)=\int_{R_{\eta}^{d}} \rho\left(x-\frac{\hbar}{2} \eta, x+\frac{\hbar}{2} \eta, \beta\right) e^{i \eta \cdot p} d \eta,
$$

where the new independent variable $p$ is the momentum. Applying the transformation (13) to the Bloch equation (8) yields the Bloch equation for the thermal equilibrium Wigner function

$$
\begin{gathered}
\partial_{\beta} w_{e}=\frac{\hbar^{2}}{8 m} \Delta_{x} w_{e}-\frac{|p|^{2}}{2 m} w_{e}-\frac{1}{2}\left[V\left(x+\frac{\hbar}{2 i} \nabla_{p}\right)+V\left(x-\frac{\hbar}{2 i} \nabla_{p}\right)\right] w_{e}-\phi w_{e} \\
w_{e}(x, p, \beta=0)=\hbar^{-d}
\end{gathered}
$$

The operators $V\left(x \pm \frac{\hbar}{2 i} \nabla_{p}\right)$ are defined as

$$
V\left(x \pm \frac{\hbar}{2 i} \nabla_{p}\right) w_{e}(x, p, \beta)=(2 \pi)^{-d} \int_{R_{\eta}^{d}} \int_{R_{q}^{d}} V\left(x \pm \frac{\hbar}{2} \eta\right) w_{e}(x, q, \beta) e^{i \eta \cdot(p-q)} d q d \eta .
$$

In the Wigner representation the thermal equilibrium particle, momentum, and momentum flux densities are

$$
\begin{gathered}
n_{e}(x)=\int_{R_{p}^{d}} w_{e}\left(x, p, \beta^{*}\right) d p, \\
\pi_{e}(x)=\int_{R_{p}^{d}} p w_{e}\left(x, p, \beta^{*}\right) d p, \\
\Pi_{e}(x)=\frac{1}{m} \int_{R_{p}^{d}} p p^{T} w_{e}\left(x, p, \beta^{*}\right) d p .
\end{gathered}
$$

The relationship between the quantum mechanical description and the classical picture is transparent using the Wigner representation. Setting $\hbar$ to zero formally in the Bloch equation (14) immediately gives the classical Maxwellian

$$
w_{e}(x, p, \beta)=\hbar^{-d} \exp \left\{-\beta\left(\frac{|p|^{2}}{2 m}+V+\phi\right)\right\} .
$$

The Fermi level $\phi$ just plays the role of a scaling parameter and is chosen to determine the total number $N_{\text {particles }}$ of particles in the system

$$
\int_{R_{x}^{d}} n_{e}(x) d x=\sum_{\lambda} \exp \left\{-\beta^{*}\left(E_{\lambda}+\phi\right)\right\}=N_{\text {particles }}
$$


Note that because of the properties of the exponential function in Boltzmann statistics, the effect of the Fermi level is to scale all quantities by the factor $\exp \left\{-\beta^{*} \phi\right\}$. This scaling can be performed after solving the Bloch equation with $\phi=0$. The situation becomes more complex for Fermi-Dirac or Bose-Einstein statistics [13].

We will construct approximate solutions to the Bloch equation (8) or its Wigner transformed version (14). The motivation for this work is threefold. (i) Transient solutions of quantum kinetic equations usually require a thermal equilibrium Wigner function as initial data. To obtain the thermal equilibrium solution either via the eigenvalue problem for the Schrödinger equation [10] or via the Bloch equation [12] is an expensive computational task. (ii) Approximations to the thermal equilibrium distribution function can be used to derive quantum mechanical corrections for macroscopic fluid dynamical equations. As in the classical case, equations for the nonequilibrium densities of particles, momentum, and energy can be derived by building the appropriate moments of the nonequilibrium quantum transport equation, the transient quantum Liouville equation. Closure of the infinite hierarchy of moment equations is obtained by assuming that the nonequilibrium Wigner function is close to the momentum-displaced thermal equilibrium Wigner function. This procedure leads to the quantum hydrodynamic (QHD) model, which includes quantum mechanical correction terms to the compressible Euler equations. (iii) The "effective" partition function $Z=\int_{R_{x}^{d}} \rho(x, x, \beta) d x$ derived from the thermal equilibrium density matrix is the starting point for solving problems in quantum statistical mechanics.

Wigner [14] derived an approximate thermal equilibrium solution in 1932:

$$
\begin{gathered}
w_{e}\left(x, p, \beta^{*}\right)=\hbar^{-d} \exp \left\{-\beta^{*}\left(\frac{|p|^{2}}{2 m}+V+\phi\right)\right\} \\
\times\left[1+\hbar^{2}\left\{-\frac{\beta^{* 2}}{8 m} \Delta_{x} V+\frac{\beta^{* 3}}{24 m}\left|\nabla_{x} V\right|^{2}+\frac{\beta^{* 3}}{24 m^{2}} p_{j} p_{k} \partial_{x_{j k}}^{2} V\right\}\right] .
\end{gathered}
$$

The corresponding QHD model has been investigated in [5]. This $O\left(\hbar^{2}\right)$ QHD model exhibits complex quantum phenomena including quantum tunneling and resonance in the resonant tunneling diode, and is an inexpensive way to simulate these phenomena compared with a solution of the full quantum Liouville equation [11, 9]. Alternative approximations to the thermal equilibrium free energy and density matrix can be found in [4] and [3], respectively. One of the drawbacks of the $O\left(\hbar^{2}\right)$ QHD equations is the appearance of second derivatives of the potential $V$ in the approximation (22) and consequently in the quantum correction to the stress tensor and energy density. Since the potential will be discontinuous at heterojunctions, this casts doubt on the validity of the $O\left(\hbar^{2}\right)$ QHD equations near potential discontinuities.

We will take a different approach to the derivation of approximate solutions to the Bloch equation based on an asymptotic expansion of the solution for "small" potentials. The main result is that in the corresponding "smooth" QHD model the potential $V$ is replaced by a smoothed potential $S\left(\frac{1}{i} \nabla_{x}\right) V$, where the symbol $S(\xi)$ of the pseudodifferential operator $S$ behaves like $|\xi|^{-2}$ for large $\xi$. This approximation is better suited for dealing with potential discontinuities and incorporates the nonlocal effects of potential barriers observed in solutions of the full quantum kinetic equations [11]. In the QHD equations the stress tensor based on the smoothed potential actually cancels the leading singularity in the classical potential at a barrier and leaves a residual smooth effective potential with a lower potential height in the barrier 
region [7]. The smoothing makes the barrier partially transparent to the particle flow and provides the mechanism for particle tunneling in the smooth QHD model.

The paper is organized as follows. In section 2 we first derive Wigner's approximate solution (22) in order to analyze its asymptotic validity. Then we proceed to derive the improved approximation based on the smoothed potential $S V$. Section 3 deals with the asymptotic validity of the smoothed potential approximation for small potentials and high temperatures. We demonstrate the convergence of the approximate Wigner function as well as the particle density. In section 4 we derive the corresponding QHD model. Section 5 is devoted to the numerical verification of the asymptotic results. It turns out that, at least for the double barrier structure considered in section 5, the parameter range for which the approximation is valid is actually much larger than expected.

Our paper [7] presents a derivation and interpretation of the smooth QHD model from a more physical viewpoint.

2. Asymptotics. We start by bringing the initial value problem (14) and (15) into an appropriate scaled and dimensionless form. Note that adding a constant to the potential $V$ should not change the overall problem and that the Fermi level $\phi$ can be eliminated from the Bloch equation. For the independent variables $x, p$, and $\beta$, we use the scaling

$$
x=L x_{s}, \quad p=\sqrt{\frac{m}{\beta^{*}}} p_{s}, \quad \beta=\beta^{*} \beta_{s},
$$

where $L$ denotes a characteristic length scale. We scale the potential by

$$
V(x)=V_{0}+\frac{\varepsilon}{\beta^{*}} V_{s}\left(x_{s}\right), \quad V_{0}=\frac{1}{2}\left[\sup _{x \in R^{d}} V+\inf _{x \in R^{d}} V\right] .
$$

An additive constant in the potential $V$ is absorbed into $V_{0}$ and does not affect $V_{s} . \varepsilon$ is a dimensionless parameter measuring the size of the potential. The scaled thermal equilibrium Wigner function and particle density are

$$
\begin{gathered}
w_{e}(x, p, \beta)=\hbar^{-d} \exp \left\{-\beta\left(V_{0}+\phi\right)\right\} w_{s}\left(x_{s}, p_{s}, \beta_{s}\right), \\
n_{e}(x)=\left(\frac{m}{\beta^{*} \hbar^{2}}\right)^{\frac{d}{2}} \exp \left\{-\beta^{*}\left(V_{0}+\phi\right)\right\} n_{s}\left(x_{s}\right) .
\end{gathered}
$$

The normalization condition (1) now reads

$$
\int_{R_{x}^{d}} n_{s}\left(x_{s}\right) d x_{s}=h^{d} \exp \left\{\beta^{*}\left(V_{0}+\phi\right)\right\}, \quad n_{s}\left(x_{s}\right)=\int_{R_{p}^{d}} w_{s}\left(x_{s}, p_{s}, 1\right) d p_{s},
$$

where here $h$ denotes the scaled Planck constant

$$
h=\hbar \sqrt{\frac{\beta^{*}}{m L^{2}}} .
$$

With this scaling the initial value problem (14) and (15) becomes

$$
\partial_{\beta_{s}} w_{s}=\frac{h^{2}}{8} \Delta_{x_{s}} w_{s}-\frac{|p|^{2}}{2} w_{s}-\frac{\varepsilon}{2}\left[V_{s}\left(x_{s}+\frac{h}{2 i} \nabla_{p_{s}}\right)+V_{s}\left(x_{s}-\frac{h}{2 i} \nabla_{p_{s}}\right)\right] w_{s},
$$




$$
w_{s}\left(x_{s}, p_{s}, 0\right)=1
$$

with $0 \leq \beta \leq 1$. The initial value problem (29) and (30) is independent of the Fermi level $\phi$, and $\phi$ can be computed after the fact from (27). After computing $\phi$ the original thermal equilibrium Wigner function can be reconstructed from (25). For notational convenience we will drop the subscript $s$ from here on.

We will first derive Wigner's approximate solution. Our derivation differs from Wigner's and illustrates the range of validity of this approximation and its limitations. We expand the operator on the right-hand side of (29) in powers of $h$ to obtain

$$
\partial_{\beta} w=-\left(\frac{|p|^{2}}{2}+\varepsilon V\right) w+\frac{h^{2}}{8}\left[\Delta_{x} w+\varepsilon\left(\partial_{x_{j} x_{k}}^{2} V\right) \partial_{p_{j} p_{k}}^{2} w\right]-\frac{\varepsilon h^{4}}{8} r_{h}\left(x, \frac{h}{2 i} \nabla_{p}\right) w,
$$

where the symbol of the remainder term $r_{h}$ is

$$
r_{h}\left(x, \frac{h}{2} \eta\right)=\frac{1}{h^{2}}\left[\delta_{h}^{2} V\left(x, \frac{h}{2} \eta\right)-\eta_{j} \eta_{k} \partial_{x_{j} x_{k}}^{2} V\right]
$$

with

$$
\delta_{h}^{2} V\left(x, \frac{h}{2} \eta\right)=\frac{4}{h^{2}}\left[V\left(x+\frac{h}{2} \eta\right)-2 V(x)+V\left(x-\frac{h}{2} \eta\right)\right] .
$$

If the potential $V$ is sufficiently smooth, the solution $w$ can be expanded in powers of $h^{2}$ :

$$
w=w_{0}+h^{2} w_{2}+h^{4} w_{4}+\cdots .
$$

Matching powers of $h^{2}$ in the Bloch equation, we obtain

$$
\partial_{\beta} w_{2}=-\left(\frac{|p|^{2}}{2}+\varepsilon V\right) w_{2}+\frac{1}{8}\left[\Delta_{x} w_{0}+\varepsilon\left(\partial_{x_{j} x_{k}}^{2} V\right) \partial_{p_{j} p_{k}}^{2} w_{0}\right], \quad w_{2}(x, p, 0)=0,
$$

yielding

$$
\begin{gathered}
w_{0}(x, p, \beta)=\exp \left\{-\beta\left(\frac{|p|^{2}}{2}+\varepsilon V\right)\right\}, \\
w_{2}(x, p, \beta)=\frac{1}{8} \exp \left\{-\beta\left(\frac{|p|^{2}}{2}+\varepsilon V\right)\right\} \\
\times\left(-\varepsilon \beta^{2} \Delta_{x} V+\frac{\varepsilon^{2} \beta^{3}}{3}\left|\nabla_{x} V\right|^{2}+\frac{\varepsilon \beta^{3}}{3} p_{j} p_{k} \partial_{x_{j} x_{k}}^{2} V\right), \\
w(x, p, 1)=\exp \left\{-\frac{|p|^{2}}{2}-\varepsilon V\right\}
\end{gathered}
$$




$$
\times\left[1+\frac{h^{2}}{8}\left(-\varepsilon \Delta_{x} V+\frac{\varepsilon^{2}}{3}\left|\nabla_{x} V\right|^{2}+\frac{\varepsilon}{3} p_{j} p_{k} \partial_{x_{j} x_{k}}^{2} V\right)\right]+O\left(h^{4}\right) .
$$

In order to compute the quantum corrections to the fluid dynamical model in the next section it will be necessary to calculate the thermal equilibrium stress tensor. A straightforward calculation [5] gives for the $O\left(h^{2}\right)$ approximation

$$
P_{j k}=-\frac{n}{\beta^{*}} \delta_{j k}-\frac{\hbar^{2} \beta^{*} n}{12 m} \partial_{x_{j k}}^{2} V+O\left(\hbar^{4}\right) .
$$

Note that $\pi=0$ since we are working in a frame comoving with the fluid.

There are several facts which should be pointed out here.

- The formula (39) represents an approximation for small values of the scaled Planck constant $h$, which is equivalent by (28) to large length scales or high temperatures.

- Equation (34) represents only an asymptotic expansion and not a convergent series since the computation of higher order terms involves successive differentiation.

- The remainder term $r_{h}$ in (31) is bounded independently of $h$ only if the potential is sufficiently smooth. In particular, we can expect this approximation to break down in the neighborhood of discontinuities in the potential.

We will now take a different tack and approximate the solution of the Bloch equation not for small values of $h$ but instead for applied potentials which are small compared to the inverse temperature $\beta^{*}$, that is, for small values of the parameter $\varepsilon$. Expanding the thermal equilibrium Wigner function in powers of $\varepsilon$ and matching powers of $\varepsilon$ in the Bloch equation yields

$$
\begin{gathered}
w_{\varepsilon}=\sum_{\lambda=0}^{\infty} w_{\lambda} \varepsilon^{\lambda} \\
\partial_{\beta} w_{0}=\frac{h^{2}}{8} \Delta_{x} w_{0}-\frac{|p|^{2}}{2} w_{0}, \quad w_{0}(x, p, 0)=1, \\
\partial_{\beta} w_{\lambda}=\frac{h^{2}}{8} \Delta_{x} w_{\lambda}-\frac{|p|^{2}}{2} w_{\lambda}-\mu V w_{\lambda-1}, \quad w_{\lambda}(x, p, 0)=0 \quad(\lambda=1,2, \ldots),
\end{gathered}
$$

where

$$
\mu V\left(x, \frac{h}{2 i} \nabla_{p}\right) w(x, p, \beta)=\frac{1}{2}\left[V\left(x+\frac{h}{2 i} \nabla_{p}\right)+V\left(x-\frac{h}{2 i} \nabla_{p}\right)\right] w(x, p, \beta) .
$$

The solution of (42) is

$$
w_{0}(x, p, \beta)=\exp \left\{-\frac{\beta}{2}|p|^{2}\right\} .
$$

To solve equation (43) for the first-order term $w_{1}$, we make use of the equivalency formula for autonomous pseudodifferential operators:

$$
\mu V\left(x, \frac{h}{2 i} \nabla_{p}\right) w_{0}(p, \beta)=\mu w_{0}\left(p, \frac{h}{2 i} \nabla_{x}, \beta\right) V(x),
$$


where the operator $\mu w_{0}$ is

$$
\mu w_{0}\left(p, \frac{h}{2 i} \nabla_{x}, \beta\right)=\frac{1}{2}\left[w_{0}\left(p+\frac{h}{2 i} \nabla_{x}, \beta\right)+w_{0}\left(p-\frac{h}{2 i} \nabla_{x}, \beta\right)\right] .
$$

It is more convenient to solve the Fourier transformed version of (43). To this end we denote by $\tilde{f}$ the Fourier transform of the function $f$ with respect to the position variable $x$ :

$$
\tilde{f}(\xi)=(2 \pi)^{-\frac{d}{2}} \int_{R_{x}^{d}} f(x) e^{-i \xi \cdot x} d x .
$$

After Fourier-transforming in $x$ and using (46), (43) reads, for $\lambda=1$,

$$
\partial_{\beta} \tilde{w}_{1}=-\left(\frac{h^{2}}{8}|\xi|^{2}+\frac{|p|^{2}}{2}\right) \tilde{w}_{1}-\mu w_{0}\left(p, \frac{h}{2} \xi, \beta\right) \tilde{V}(\xi), \quad \tilde{w}_{1}(\xi, p, 0)=0 .
$$

In deriving this expression we have used the fact that the zeroth-order term $w_{0}$ is actually independent of the position variable $x$. The first-order term $\tilde{w}_{1}$ in the expansion is therefore

$$
\tilde{w}_{1}(\xi, p, \beta)=-\beta g(\xi, p, \beta) \tilde{V}(\xi), \quad w_{1}(x, p, \beta)=-\beta g\left(\frac{1}{i} \nabla_{x}, p, \beta\right) V(x)
$$

with the function $g$ defined by

$$
g(\xi, p, \beta)=\frac{1}{\beta} \int_{0}^{\beta} \exp \left\{(\gamma-\beta)\left(\frac{h^{2}}{8}|\xi|^{2}+\frac{|p|^{2}}{2}\right)\right\} \mu w_{0}\left(p, \frac{h}{2} \xi, \gamma\right) d \gamma .
$$

Thus the $O(\varepsilon)$ approximation of the thermal equilibrium Wigner function is

$$
w(x, p, \beta)=\exp \left\{-\frac{\beta}{2}|p|^{2}\right\}-\varepsilon \beta g\left(\frac{1}{i} \nabla_{x}, p, \beta\right) V(x)+O\left(\varepsilon^{2}\right) .
$$

For the fluid dynamical model in section 4 we will need the corresponding approximations to the particle density and the stress tensor (again $\pi=0$ ):

$$
\begin{gathered}
n(x)=(2 \pi)^{\frac{d}{2}}\left[1-\varepsilon G_{0}\left(\frac{1}{i} \nabla_{x}\right) V(x)\right]+O\left(\varepsilon^{2}\right), \\
P(x)=-(2 \pi)^{\frac{d}{2}}\left[\mathbf{I}-\varepsilon G_{2}\left(\frac{1}{i} \nabla_{x}\right) V(x)\right]+O\left(\varepsilon^{2}\right),
\end{gathered}
$$

where the symbols of the pseudodifferential operators $G_{0}$ and $G_{2}$ are

$$
\begin{gathered}
G_{0}(\xi)=(2 \pi)^{-\frac{d}{2}} \int_{R_{p}^{d}} g(\xi, p, 1) d p \\
G_{2}(\xi)=(2 \pi)^{-\frac{d}{2}} \int_{R_{p}^{d}} p p^{T} g(\xi, p, 1) d p .
\end{gathered}
$$


The calculation of $G_{0}$ and $G_{2}$ is deferred to Appendix A. The results for the symbols of the operators $G_{0}$ and $G_{2}$ are

$$
G_{0}(\xi)=\kappa\left(\frac{h}{2}|\xi|\right)
$$

$$
G_{2}(\xi)=\frac{\xi \xi^{T}}{|\xi|^{2}}+\kappa\left(\frac{h}{2}|\xi|\right)\left[\mathbf{I}-\frac{\xi \xi^{T}}{|\xi|^{2}}\right]
$$

where

$$
\kappa(z)=\int_{0}^{1} \exp \left\{\left(\gamma^{2}-1\right) \frac{z^{2}}{2}\right\} d \gamma
$$

The thermal equilibrium stress tensor in the $O(\varepsilon)$ approximation is

$$
P=-n \mathbf{I}-\varepsilon n\left(G_{0} \mathbf{I}-G_{2}\right)\left(\frac{1}{i} \nabla_{x}\right) V+O\left(\varepsilon^{2}\right) .
$$

In analogy with the Wigner approximation $(40),(60)$ can be written as

$$
P_{j k}=-n \delta_{j k}-\frac{\varepsilon h^{2} n}{12} \partial_{x_{j k}}^{2} S_{h}\left(\frac{1}{i} \nabla_{x}\right) V+O\left(\varepsilon^{2}\right),
$$

where the symbol of the smoothing operator $S_{h}$ is

$$
S_{h}(\xi)=\frac{12}{h^{2}|\xi|^{2}}\left[1-\kappa\left(\frac{h}{2}|\xi|\right)\right] .
$$

In unscaled form, (61) and (62) read

$$
\begin{gathered}
P=-\frac{n}{\beta^{*}} \mathbf{I}-\frac{\hbar^{2} \beta^{*} n}{12 m} \partial_{x}^{\otimes 2} S_{\hbar}\left(\frac{1}{i} \nabla_{x}\right) V+O\left(\left(\beta^{*} V\right)^{2}\right), \\
S_{\hbar}(\xi)=\frac{12 m}{\hbar^{2} \beta^{*}|\xi|^{2}}\left[1-\kappa\left(\frac{\hbar}{2} \sqrt{\frac{\beta^{*}}{m}}|\xi|\right)\right] .
\end{gathered}
$$

Simple Taylor expansion implies that $S_{h}(\xi)=1+O\left(h^{2}\right)$ for $h \rightarrow 0$. In this sense the approximation (63) is consistent with the Wigner approximation (40). However, for large values of $\xi, S_{h}(\xi)$ behaves like $|\xi|^{-2}$ and the quantum correction $\partial_{x}^{2} S V$ has the same degree of smoothness as the original potential $V$. In fact, the stress tensor (63) actually cancels the leading singularity in the classical potential at a barrier and leaves a residual smooth effective potential with a lower potential height in the barrier region [7].

One disturbing fact remains: the approximation (52) of the thermal equilibrium Wigner function does not reduce to the classical Maxwellian

$$
\exp \left\{-\frac{\beta}{2}|p|^{2}-\varepsilon \beta V\right\}
$$


in the classical limit $h \rightarrow 0$. Letting $h \rightarrow 0$ in (52) yields

$$
\begin{gathered}
\lim _{h \rightarrow 0} g(\xi, p, \beta)=\exp \left\{-\frac{\beta}{2}|p|^{2}\right\}, \\
\lim _{h \rightarrow 0}\left(w_{0}+\varepsilon w_{1}\right)=\exp \left\{-\frac{\beta}{2}|p|^{2}\right\}[1-\varepsilon \beta V(x)],
\end{gathered}
$$

which is only the $O(\varepsilon)$ approximation to the Maxwellian. This discrepancy can be remedied by replacing terms of the form $1-\varepsilon z$ by $e^{-\varepsilon z}$ in the formulas (52)-(54), thereby introducing only an additional $O\left(\varepsilon^{2}\right)$ error term:

$$
\begin{gathered}
w(x, p, \beta)=\exp \left\{-\frac{\beta}{2}|p|^{2}-\varepsilon \beta g_{1}\left(\frac{1}{i} \nabla_{x}, p, \beta\right) V(x)\right\}+O\left(\varepsilon^{2}\right), \\
n(x)=(2 \pi)^{\frac{d}{2}} \exp \left\{-\varepsilon G_{0}\left(\frac{1}{i} \nabla_{x}\right) V(x)\right\}+O\left(\varepsilon^{2}\right), \\
P_{j k}(x)=\left\{\begin{array}{cc}
-(2 \pi)^{\frac{d}{2}} \exp \left\{-\varepsilon G_{2_{j k}}\left(\frac{1}{i} \nabla_{x}\right) V(x)\right\}+O\left(\varepsilon^{2}\right), & j=k, \\
(2 \pi)^{\frac{d}{2}} \varepsilon G_{2_{j k}}\left(\frac{1}{i} \nabla_{x}\right) V(x)+O\left(\varepsilon^{2}\right), & j \neq k,
\end{array}\right.
\end{gathered}
$$

with $g_{1}(\xi, p, \beta)=\exp \left\{\beta|p|^{2} / 2\right\} g(\xi, p, \beta)$. The approximation (68) now reduces to the classical Maxwellian for $\varepsilon \rightarrow 0$ as well as for $h \rightarrow 0$. Since the difference between (68) and (52) is $O\left(\varepsilon^{2}\right),(70)$ yields the same stress tensor (63).

3. Convergence. We now analyze the asymptotic validity of the expansion derived in section 2 by providing estimates of the remainder term. We define the remainder term $r$ by $w=w_{1}+\varepsilon w_{2}+\varepsilon^{2} r$ and obtain

$$
\partial_{\beta} r=\frac{h^{2}}{8} \Delta_{x} r-\frac{|p|^{2}}{2} r-\mu V\left(x, \frac{h}{2 i} \nabla_{p}\right)\left[w_{1}+\varepsilon r\right], \quad r(x, p, 0)=0 .
$$

To prove the boundedness of the remainder term $r$ in the $L^{2}\left(R_{x}^{d} \times R_{p}^{d}\right)$ norm is a straightforward exercise in energy inequalities. However, we are more concerned with the approximation quality of the zeroth- and second-order moments of the expansion, i.e., with the convergence of the particle density and the momentum flux density tensor. The main result of this section is Theorem 3, which proves the convergence of the particle density as $\varepsilon \rightarrow 0$. The convergence of the momentum flux density tensor remains an unresolved problem at this point, although the numerical evidence in section 5 indicates that the smoothed potential approximation also produces a good approximation to $\Pi$. We start with

TheOrem 1. Let the potential $V$ be bounded. Then

$$
\max _{0 \leq \beta \leq 1}\left\{\|r(\beta)\|_{L^{2}\left(R_{x}^{d} \times R_{p}^{d}\right)}\right\} \leq \mathrm{const}\|V\|_{L^{\infty}\left(R_{x}^{d}\right)} \max _{0 \leq \beta \leq 1}\left\{\left\|w_{1}(\beta)\right\|_{L^{2}\left(R_{x}^{d} \times R_{p}^{d}\right)}\right\},
$$

where const denotes a positive constant independent of $\varepsilon$ and $h$.

Proof. Multiplying both sides of (71) by $r$ and integrating with respect to $x$ and $p$ gives

$$
\frac{1}{2} \partial_{\beta}\|r(x, p, \beta)\|_{L^{2}\left(R_{x}^{d} \times R_{p}^{d}\right)}=-\int_{R_{p}^{d}} \int_{R_{x}^{d}}\left(\frac{h^{2}}{8}\left|\nabla_{x} r\right|^{2}+\frac{1}{2}|p r|^{2}+r \mu V\left[\varepsilon r+w_{1}\right]\right) d x d p .
$$


Using the definition of the pseudodifferential operator $\mu V$ yields

$$
\begin{gathered}
\int_{R_{p}^{d}} \int_{R_{x}^{d}} r \mu V\left[\varepsilon r+w_{1}\right] d x d p=\int_{R_{\eta}^{d}} \int_{R_{x}^{d}} \tilde{r}^{*}(x, \eta, \beta) \mu V\left(x, \frac{h}{2} \eta\right)\left[\varepsilon \tilde{r}+\tilde{w}_{1}\right] d x d \eta \\
\leq\|V\|_{L^{\infty}\left(R_{x}^{d}\right)}\|r\|_{L^{2}\left(R_{x}^{d} \times R_{p}^{d}\right)}\left\|\varepsilon r+w_{1}\right\|_{L^{2}\left(R_{x}^{d} \times R_{p}^{d}\right)} .
\end{gathered}
$$

Combining (73) with (74) implies

$$
\partial_{\beta}\|r\|_{L^{2}\left(R_{x}^{d} \times R_{p}^{d}\right)} \leq \text { const }\|V\|_{L^{\infty}\left(R_{x}^{d}\right)}\left[\varepsilon\|r\|_{L^{2}\left(R_{x}^{d} \times R_{p}^{d}\right)}+\left\|w_{1}\right\|_{L^{2}\left(R_{x}^{d} \times R_{p}^{d}\right)}\right] .
$$

The more interesting question, however, is the convergence of the moments of the remainder term, i.e., the degree of approximation of the particle and momentum flux densities. The existence of the moments of the Wigner function in the presence of discontinuous potentials is a challenging problem (see $[1,2]$ for the treatment of the transient case). In the thermal equilibrium case described by the Bloch equation, we are aided by the smoothing properties of the operator $h^{2} \Delta_{x} / 8-|p|^{2} / 2$.

Lemma 1. For a function $f(x, p)$ let the $\lambda$ th-order moment $M_{\lambda} f(x)$ be defined by

$$
M_{\lambda} f(x)=\int_{R_{p}^{d}} p^{\otimes \lambda} f(x, p) d p, \quad \lambda=0,1,2, \ldots
$$

Then

$$
\left\|M_{\lambda} f\right\|_{L^{2}\left(R_{x}^{d}\right)} \leq \mathrm{const}\left\|\left(1+|p|^{2}\right)^{\frac{\alpha}{2}} f(x, p)\right\|_{L^{2}\left(R_{x}^{d} \times R_{p}^{d}\right)}
$$

for $\alpha>\lambda+\frac{1}{2}$.

Proof. We have

$$
\begin{gathered}
\left\|M_{\lambda} f\right\|_{L^{2}\left(R_{x}^{d}\right)}^{2}=\int_{R_{x}^{d}}\left[\int_{R_{p}^{d}} p^{\otimes \lambda} f(x, p) d p\right]^{2} d x \\
\leq \int_{R_{x}^{d}}\left[\int_{R_{p}^{d}}\left|p^{\otimes \lambda}\right|^{2}\left(1+|p|^{2}\right)^{-\alpha} d p\right]\left[\int_{R_{p}^{d}}\left(1+|p|^{2}\right)^{\alpha}|f(x, p)|^{2} d p\right] d x .
\end{gathered}
$$

For $\alpha>\lambda+\frac{1}{2}$ the term $\int_{R_{p}^{d}}\left|p^{\otimes \lambda}\right|^{2}\left(1+|p|^{2}\right)^{-\alpha} d p$ remains bounded and the result follows immediately.

In order to estimate the moments of the remainder term $r$, we will estimate the $L^{2}$-norm of the function $r_{\alpha}$ defined by

$$
r_{\alpha}(x, p, \beta)=\left(1+|p|^{2}\right)^{\frac{\alpha}{2}} r(x, p, \beta) .
$$

$r_{\alpha}$ satisfies the initial value problem

$$
\partial_{\beta} r_{\alpha}=\frac{h^{2}}{8} \Delta_{x} r_{\alpha}-\frac{|p|^{2}}{2} r_{\alpha}-\Omega_{\alpha}\left[w_{1 \alpha}+\varepsilon r_{\alpha}\right], \quad r_{\alpha}(x, p, 0)=0,
$$

where

$$
\Omega_{\alpha}=\left(1+|p|^{2}\right)^{\frac{\alpha}{2}} \mu V\left(x, \frac{h}{2 i} \nabla_{p}\right)\left(1+|p|^{2}\right)^{-\frac{\alpha}{2}}
$$


and

$$
w_{1 \alpha}(x, p, \beta)=\left(1+|p|^{2}\right)^{\frac{\alpha}{2}} w_{1}(x, p, \beta) .
$$

For the estimate of $r_{\alpha}$ we will use a specific norm defined by

$$
\|f(x, p, \beta)\|^{2}=\int_{R_{p}^{d}} \int_{R_{x}^{d}} \max _{0 \leq \beta \leq 1}\left\{|\tilde{f}(\xi, p, \beta)|^{2}\right\} d x d p,
$$

where

$$
\tilde{f}(\xi, p, \beta)=(2 \pi)^{-\frac{d}{2}} \int_{R_{x}^{d}} f(x, p, \beta) e^{-i \xi \cdot x} d x .
$$

In this norm we can estimate the solution of the initial value problem (71) with the following theorem.

THEOREM 2. Let the function $r$ satisfy the initial value problem

$$
\partial_{\beta} r=\frac{h^{2}}{8} \Delta_{x} r-\frac{|p|^{2}}{2} r-\Omega_{\alpha}\left[\varepsilon r+w_{1 \alpha}\right], \quad 0 \leq \beta \leq 1, \quad r(x, p, 0)=0
$$

with the operator $\Omega_{\alpha}$ defined in (81). Let the potential $V$ be smooth enough that its Fourier transform satisfies $|\tilde{V}(\xi)|^{2} \leq$ const $\left[1+|\xi|^{2}\right]^{-1}$. Then for $\varepsilon$ sufficiently small, $\|r\| \leq$ const $\left\|w_{1 \alpha}\right\|$ for $0 \leq \alpha<3$.

The proof is deferred to Appendix B. The condition on the smoothness of the potential in Theorem 2 is modest and allows for discontinuous potentials. What remains to be shown is the bound on the inhomogeneous term $w_{1 \alpha}$ in (80). Unfortunately, $w_{1 \alpha}$ is bounded in the norm defined in (83) only for $0 \leq \alpha<3 / 2$. While this range of $\alpha$ provides a bound for the zeroth-order moment (the particle density), a value $\alpha>5 / 2$ would be needed to estimate the second-order moment (the momentum flux density tensor).

Lemma 2. Let the function $w_{1 \alpha}$ be defined by

$$
w_{1 \alpha}(x, p, \beta)=\left(1+|p|^{2}\right)^{\frac{\alpha}{2}} w_{1}(x, p, \beta)
$$

with $w_{1}$ given by (50). Then $\left\|w_{1 \alpha}\right\|<\infty$ for $0 \leq \alpha<3 / 2$ with the norm defined in (83).

The proof is deferred to Appendix C.

Combining Theorem 2 and Lemma 2 produces the bound on the function $r_{\alpha}$.

THEOREM 3. Let the remainder term $r$ and the function $r_{\alpha}$ be the solutions of the initial value problems (71) and (80), respectively. Then $\left\|r_{\alpha}\right\| \leq$ const for $0 \leq \alpha<\frac{3}{2}$. Consequently, $\left\|M_{0} r\right\|_{L^{2}\left(R_{x}^{d}\right)} \leq$ const by Lemma 1, and the approximation of the particle density in section 2 satisfies

$$
\left\|n-n_{0}-\varepsilon n_{1}\right\|_{L^{2}\left(R_{x}^{d}\right)}=O\left(\varepsilon^{2}\right) .
$$

4. QHD models. Next we discuss the QHD models corresponding to the approximations of the thermal equilibrium distribution function derived in section 2 . The nonequilibrium Wigner function is the solution of the quantum Liouville (or Wigner-Boltzmann) equation

$$
\partial_{t} f+\frac{1}{m} \operatorname{div}_{x}(p f)-\theta f=C f,
$$


where the operator $\theta$ is defined by

$$
\theta=\frac{i}{\hbar}\left[V\left(x+\frac{\hbar}{2 i} \nabla_{p}\right)-V\left(x-\frac{\hbar}{2 i} \nabla_{p}\right)\right] .
$$

The quantum Liouville equation can be derived from the transient Schrödinger equation in the same way the Bloch equation is derived from the eigenvalue problem for the Schrödinger equation. The derivation makes use of the transformation

$$
f(x, p, t)=\sum_{\lambda} a_{\lambda} \int_{R_{\eta}^{d}} \psi_{\lambda}^{*}\left(x+\frac{\hbar}{2} \eta, t\right) \psi_{\lambda}\left(x-\frac{\hbar}{2} \eta, t\right) e^{i \eta \cdot p} d \eta
$$

where the wavefunctions $\psi_{\lambda}(x, t)$ are solutions of the transient Schrödinger equation and the $a_{\lambda}$ 's are occupation numbers: $\sum_{\lambda} a_{\lambda}=N_{\text {particles. }}$. The collision operator $C$ in (88) is added after the fact and its form is usually based on some ad hoc assumptions. (Typically either a relaxation time model or a Fokker-Planck collision operator is assumed.) We will leave the form of the collision operator open.

We denote the average value of a quantity $\chi$ with the distribution function $f$ by

$$
\langle\chi\rangle(x, t)=\int_{R_{p}^{d}} \chi(x, p, t) f(x, p, t) d p .
$$

Integrating the quantum Liouville equation against $1, p$, and $|p|^{2} / 2 m$ yields conservation laws for particle number, momentum, and energy:

$$
\begin{gathered}
\partial_{t}\langle 1\rangle+\frac{1}{m} \operatorname{div}_{x}\langle p\rangle=\langle C\rangle \equiv C_{0}, \\
\partial_{t}\langle p\rangle+\operatorname{div}_{x}\left\langle\frac{p p^{T}}{m}\right\rangle+\langle 1\rangle \nabla_{x} V=\langle p C\rangle \equiv C_{1}, \\
\partial_{t}\left\langle\frac{|p|^{2}}{2 m}\right\rangle+\operatorname{div}_{x}\left\langle\frac{|p|^{2} p}{2 m^{2}}\right\rangle+\left\langle\frac{p}{m}\right\rangle \cdot \nabla_{x} V=\left\langle\frac{|p|^{2}}{2 m} C\right\rangle \equiv C_{2} .
\end{gathered}
$$

The system (92)-(94) has to be closed by expressing $\Pi=\left\langle p p^{T} / m\right\rangle$ and $\Theta \equiv\left\langle|p|^{2} p / 2 m^{2}\right\rangle$ in terms of (say) the conserved quantities $n=\langle 1\rangle, \pi=\langle p\rangle$, and $W=\left\langle|p|^{2} / 2 m\right\rangle$. Note that the moment equations are identical to those for the classical Boltzmann transport equation and that quantum effects enter through the closure conditions.

We first change from the moments with respect to $p$ to the centered moments with respect to the variable $p^{\prime}$ defined by

$$
p=p^{\prime}+m u, \quad\langle p\rangle=m n u,
$$

where $u$ denotes the macroscopic fluid velocity. This shift gives the transformations

$$
\begin{gathered}
\Pi=\left\langle\frac{p p^{T}}{m}\right\rangle=m n u u^{T}+\left\langle\frac{p^{\prime} p^{T}}{m}\right\rangle=m n u u^{T}-P, \\
W=\left\langle\frac{|p|^{2}}{2 m}\right\rangle=\frac{1}{2} m n|u|^{2}+\left\langle\frac{\left|p^{\prime}\right|^{2}}{2 m}\right\rangle=\frac{1}{2} m n|u|^{2}-\frac{1}{2} \operatorname{Trace}\{P\},
\end{gathered}
$$


$\Theta=\left\langle\frac{|p|^{2} p}{2 m^{2}}\right\rangle=u\left(\left\langle\frac{\left|p^{\prime}\right|^{2}}{2 m}\right\rangle+\frac{1}{2} m n|u|^{2}\right)+u^{T}\left\langle\frac{p^{\prime} p^{\prime T}}{m}\right\rangle+\left\langle\frac{\left|p^{\prime}\right|^{2} p^{\prime}}{2 m^{2}}\right\rangle=u W-u^{T} P+q$,

where $q$ is the heat flux vector. We obtain

$$
\begin{gathered}
\partial_{t} n+\operatorname{div}_{x}(n u)=C_{0}, \\
\partial_{t}(m n u)+\operatorname{div}_{x}\left(m n u u^{T}-P\right)+n \nabla_{x} V=C_{1}, \\
\partial_{t} W+\operatorname{div}_{x}\left(u W-u^{T} P+q\right)+n u \cdot \nabla_{x} V=C_{2} .
\end{gathered}
$$

Closure is obtained by assuming that the distribution function $f$ is close to a momentum-displaced thermal equilibrium distribution. We assume that

$$
\begin{gathered}
f(x, p, t)=A(x, t) w_{e}(x, p-m u), \\
\left\langle p^{\prime \otimes \lambda}\right\rangle=A(x, t) \int_{R_{p}^{d}} p^{\otimes \lambda} w_{e}(x, p) d p
\end{gathered}
$$

for a smooth function $A(x, t)$. We will use (103) to express $P, W$, and $q$ in terms of the variables $n, u$, and $T$, where $\beta^{*}$ is replaced by $1 / T$ with $T$ the local temperature. In the classical case the thermal equilibrium distribution function is the Maxwellian $\exp \left\{-|p|^{2} / 2 m T\right\}$, which yields

$$
\begin{gathered}
P=-n T \mathbf{I}, \\
W=\frac{3}{2} n T+\frac{1}{2} m n|u|^{2},
\end{gathered}
$$

and leads to the classical compressible Euler equations. For the Wigner approximation (39) and the smoothed potential approximation (68), the stress tensor $P$ is calculated in (40) and (63), respectively. The Wigner approximation yields the $O\left(\hbar^{2}\right)$ quantum hydrodynamic model treated in [5]. For the smoothed potential approximation in section 2 , we obtain from (63)

$$
\begin{gathered}
P_{j k}=-n T \delta_{j k}-\frac{\hbar^{2} n}{12 m T} \partial_{x_{j k}}^{2}(S V), \\
W=\frac{3}{2} n T+\frac{1}{2} m n|u|^{2}+\frac{\hbar^{2} n}{24 m T} \Delta_{x}(S V),
\end{gathered}
$$

where the smoothed potential $S V$ is

$$
S V(x)=(2 \pi)^{-d} \int_{R_{\xi}^{d}} \int_{R_{y}^{d}} S_{\hbar}(\xi) V(y) e^{i \xi \cdot(x-y)} d y d \xi
$$

with

$$
S_{\hbar}(\xi)=\frac{12 m T_{0}}{\hbar^{2}|\xi|^{2}}\left[1-\kappa\left(\frac{\hbar}{2} \frac{|\xi|}{\sqrt{m T_{0}}}\right)\right]
$$

and

$$
\kappa(z)=\int_{0}^{1} \exp \left\{\left(\gamma^{2}-1\right) \frac{z^{2}}{2}\right\} d \gamma
$$

Note that even though $q=0$ for both the Wigner and smoothed potential approximations, we have allowed a nonzero $q$ in the QHD equations since heat conduction plays a quantitative role in electron and hole propagation in actual semiconductor devices. 


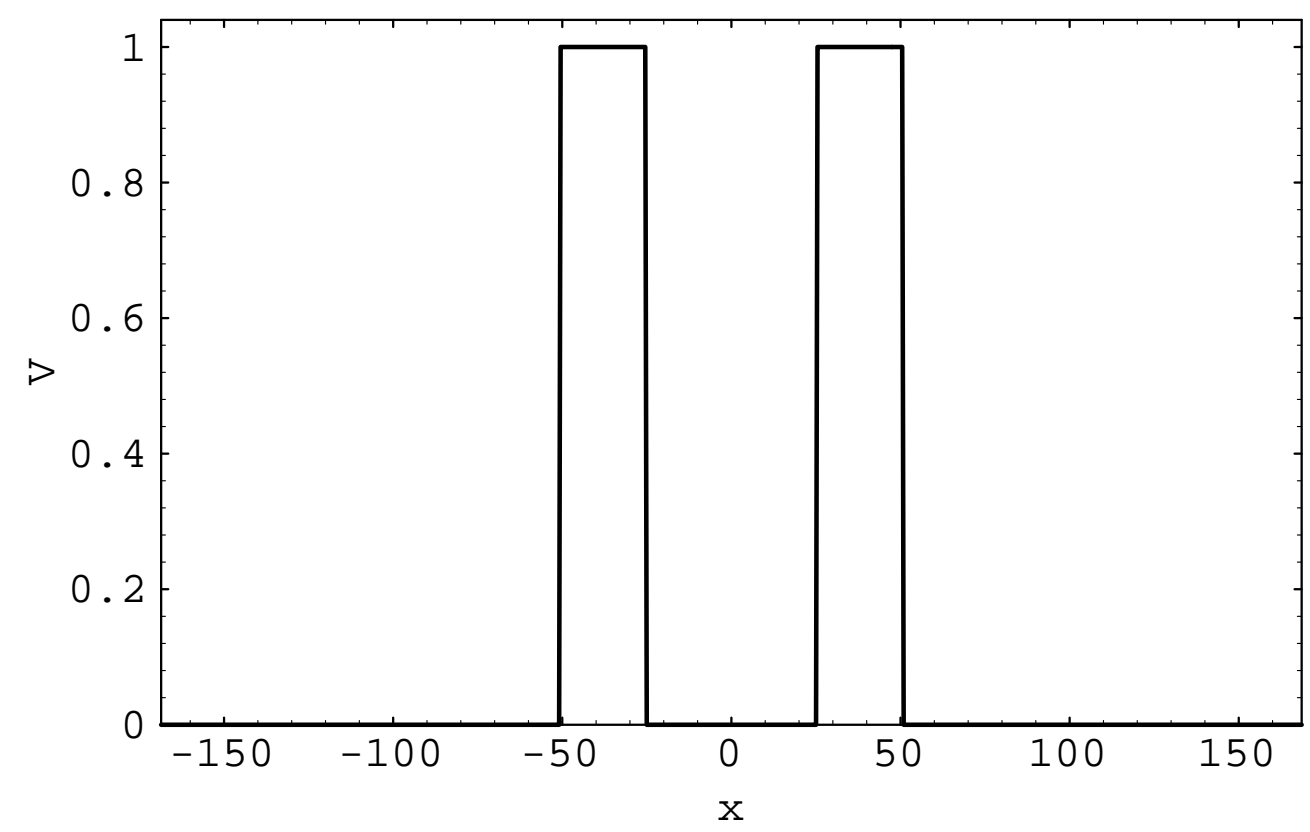

FIG. 1. Unit double barrier potential. $x$ is in $\AA$ for all figures.

5. Numerical results. In this section we present numerical results to verify the asymptotic expansions of section 2 and to test the limits of their validity. To this end we consider an electron gas in a $\mathrm{GaAs} / \mathrm{Al}_{x} \mathrm{Ga}_{1-x} \mathrm{As}$ double barrier heterostructure (see Figure 1) at $300 \mathrm{~K}$. (The double barrier heterostructure is the heart of the resonant tunneling diode.) The barrier width is $25 \AA$ and the well width is $50 \AA$ on a characteristic length scale of $100 \AA$. Technologically relevant barrier heights are in the range $0.1-0.7 \mathrm{eV}$. Without loss of generality we can assume that the barriers are perpendicular to the $x_{1}$ coordinate axis. The salient features of the numerical results are the following:

- The asymptotic expansion (68) yields a good approximation of both the particle density and the energy density. (Note that the velocity $u=0$.)

- The range of validity of the expansion extends far beyond the small $\varepsilon$ regime to $\varepsilon \approx 2-10$.

- The smoothed potential approximation for the stress tensor (63) is uniformly better than the one obtained through the Wigner approximation (40).

We will compare the Wigner and smoothed potential approximations with a numerical solution of the Bloch equation. The Bloch equation was solved on a grid of $200 \Delta x$ using the backward Euler method with homogeneous Neumann boundary conditions. A detailed description of the basic numerical method plus extensions is given in [6]. Numerical values for the constants and scaling factors in section 2 are given in Table 1.

The parameter $\varepsilon$ is chosen so that the scaled potential $V_{s}$ varies between -1 and 1. We have set the effective electron mass in GaAs to $m=0.063 m_{e}$, where $m_{e}$ is the electron mass. 
TABLE 1 by $\mathrm{eV}$.

Numerical values of constants used in the computations. $B$ denotes the barrier height divided

\begin{tabular}{|c|c|}
\hline Constant & Value \\
\hline$\hbar$ & $6.582 \times 10^{-16} \mathrm{eV} \mathrm{sec}^{-17} \mathrm{eV} \mathrm{sec}^{2} \mathrm{~cm}^{-2}$ \\
\hline$m$ & $3.582 \times 10^{-1}$ \\
\hline$\beta^{*}$ & $38.68 \mathrm{eV}^{-1}$ \\
\hline$L$ & $10^{-6} \mathrm{~cm}$ \\
\hline$h$ & 0.6840 \\
\hline$\varepsilon$ & $19.34 \mathrm{~B}$ \\
\hline
\end{tabular}

Since the potential $V$ is one-dimensional, we can reduce the number of independent variables in the Bloch equation by setting

$$
w(x, p, \beta)=\bar{w}\left(x_{1}, p_{1}, \beta\right) \exp \left\{-\frac{\beta}{2}\left(p_{2}^{2}+p_{3}^{2}\right)\right\} .
$$

The function $\bar{w}$ satisfies the initial value problem

$$
\begin{gathered}
\partial_{\beta} \bar{w}=\frac{h^{2}}{8} \partial_{x}^{2} \bar{w}-\frac{p_{1}^{2}}{2} \bar{w}-\frac{\varepsilon}{2}\left[V\left(x_{1}+\frac{h}{2 i} \partial_{p_{1}}\right)+V\left(x_{1}-\frac{h}{2 i} \partial_{p_{1}}\right)\right] \bar{w} \\
\bar{w}\left(x_{1}, p_{1}, 0\right)=1
\end{gathered}
$$

with $0 \leq \beta \leq 1$.

Figures 2-4 show the case of a barrier height of $0.01 \mathrm{eV}$, which corresponds to $\varepsilon=0.1934$. Figure 2 shows the electron density. (The overall scale for the solutions is set by requiring $\int n\left(x_{1}\right) d x_{1}=1 \mathrm{~cm}^{-2}$.) Figure 3 shows the quantum mechanical correction terms $Q_{s}$ (for the smoothed potential approximation) and $Q_{f}$ (for the numerical solution of the full Bloch equation) in the electron momentum flux:

$$
\begin{gathered}
Q_{s}=\frac{\varepsilon h^{2}}{12} \partial_{x_{1}}^{2} S_{h}\left(\frac{1}{i} \partial_{x_{1}}\right) V\left(x_{1}\right), \\
Q_{f}=-\frac{P_{11}}{n}-T .
\end{gathered}
$$

The smooth effective potential $Q+V$ for the smoothed potential approximation and for the numerical solution of the full Bloch equation is shown in Figure 4. In Figures $2-10$, the solid lines correspond to the smoothed potential approximations and the dashed lines correspond to numerical solutions of the full Bloch equation.

Figures 5-7 and 8-10 are a repetition of Figures 2-4 for higher potential barriers. Figures $5-7$ show the results for a $0.1 \mathrm{eV}$ double barrier, corresponding to $\varepsilon=1.934$, and Figures 8-10 show the results for a barrier height of $0.5 \mathrm{eV}$, corresponding to $\varepsilon=9.67$.

The electron density in Figures 2, 5, and 8 is minimized inside the potential barriers and has a local maximum at the center of the quantum well. The energy density drops precipitously as the electrons tunnel through the potential barriers and rises dramatically as the electrons exit from the barriers. The effect of the quantum correction in Figures 3,6, and 9 is to partially cancel the effects of the potential barriers (see Figures 4, 7, and 10). 


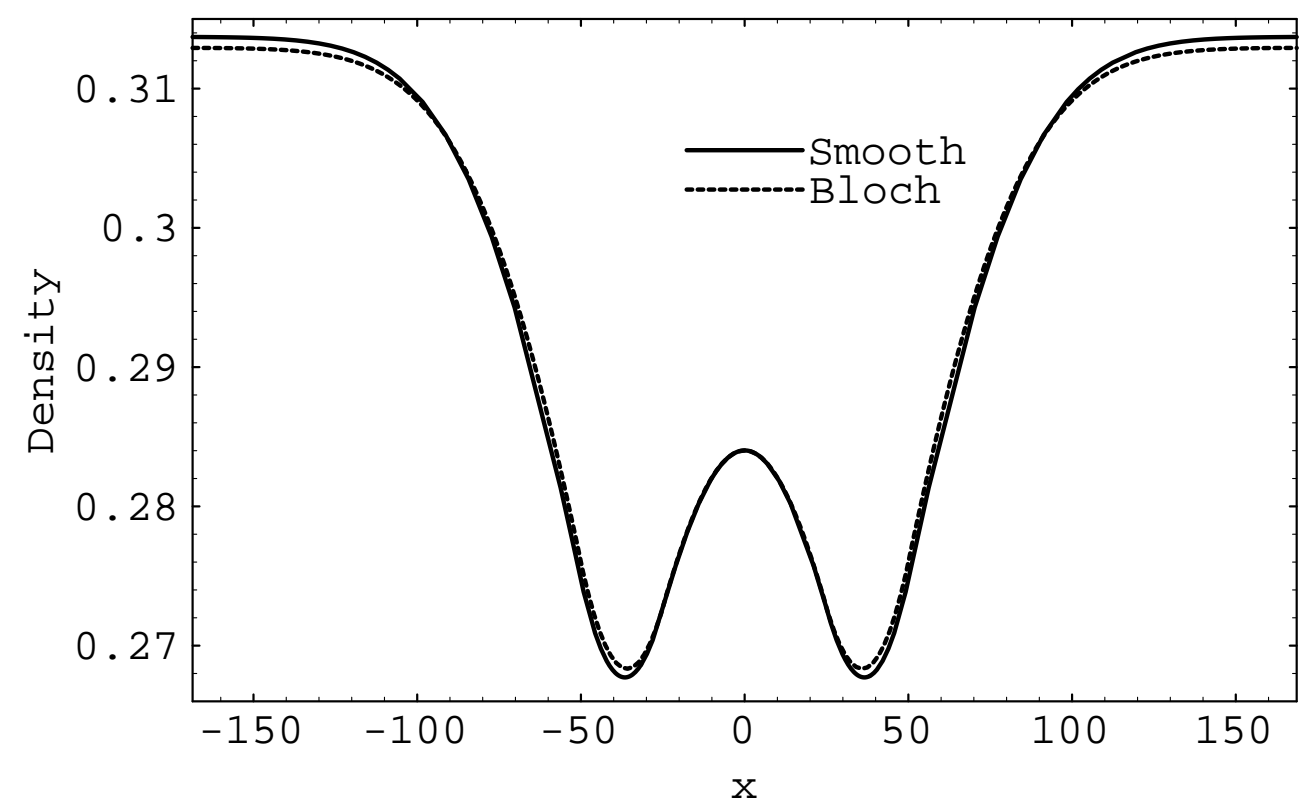

FIG. 2. Electron density in $10^{6} \mathrm{~cm}^{-3}$ for a $0.01 \mathrm{eV}$ double barrier in GaAs at $300 \mathrm{~K}(\varepsilon \approx 0.2)$. Note that the vertical axis begins at 0.266 .

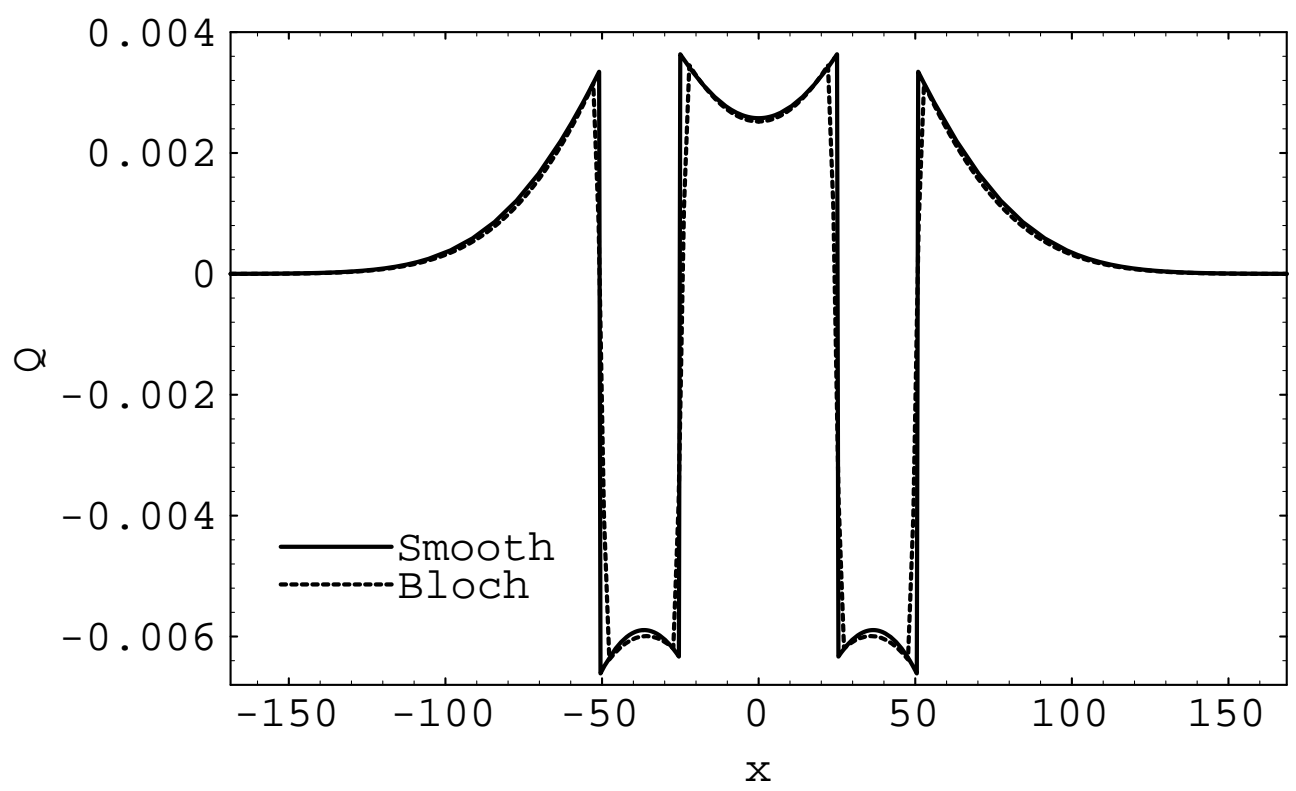

FIG. 3. Quantum correction term in $\mathrm{eV}$ for a $0.01 \mathrm{eV}$ double barrier $(\varepsilon \approx 0.2)$. 


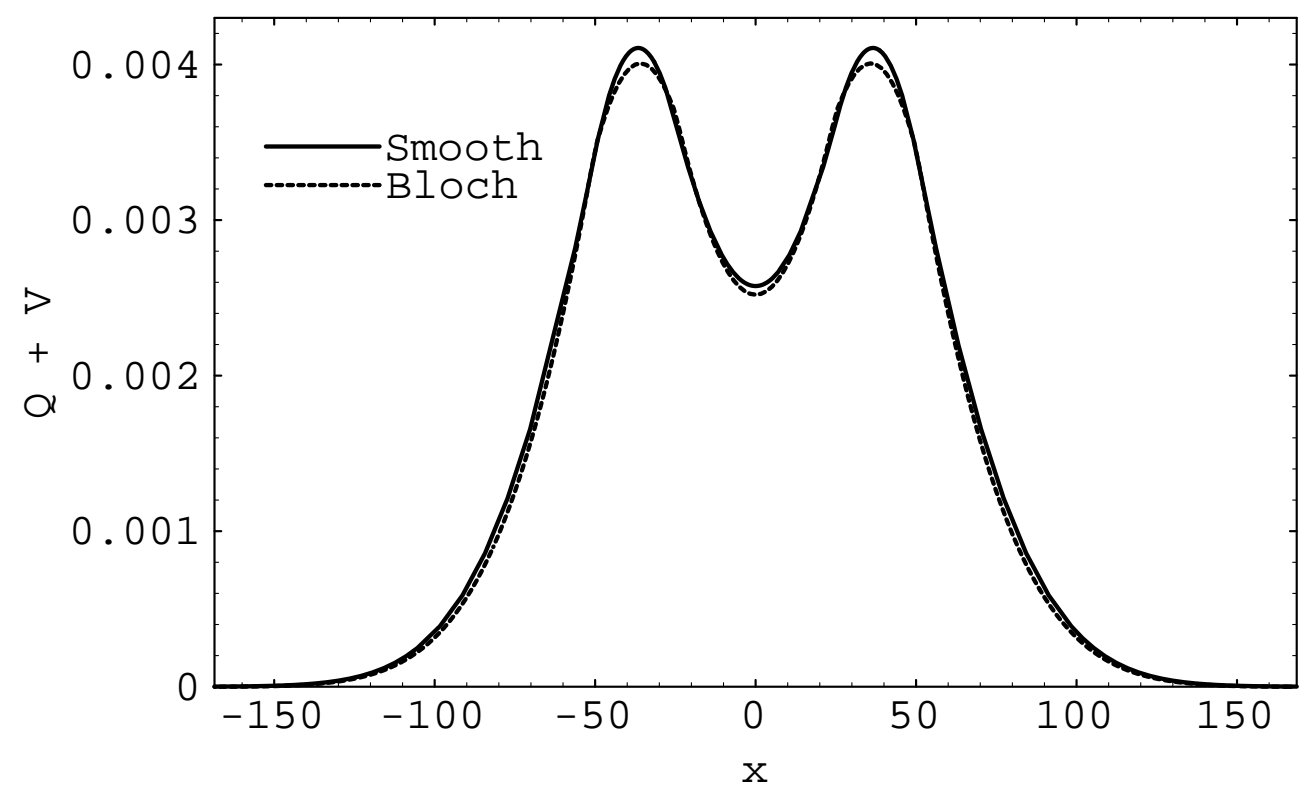

FIG. 4. Smooth effective potential in $\mathrm{eV}$ for a $0.01 \mathrm{eV}$ double barrier $(\varepsilon \approx 0.2)$.

Although the difference between the smoothed potential approximation and the "exact" solution in Figures 6 and 9 becomes significant, we still obtain a qualitatively good approximation to the quantum correction term $Q_{f}$ for large values of the perturbation parameter $\varepsilon$.

In contrast, the quantum correction term $Q_{w}$ obtained from the Wigner approximation (39) is completely dominated by the dipole behavior induced by the potential discontinuities:

$$
Q_{w}=\frac{\varepsilon h^{2}}{12} \partial_{x_{1}}^{2} V\left(x_{1}\right)=\frac{\varepsilon h^{2} B}{12}\left[\delta^{\prime}\left(x_{1}+2 a\right)-\delta^{\prime}\left(x_{1}+a\right)+\delta^{\prime}\left(x_{1}-a\right)-\delta^{\prime}\left(x_{1}-2 a\right)\right]
$$

with $a=25 \AA$. Thus our numerical evidence, convergence results, and the dipole behavior of $Q_{w}$ demonstrate that the smoothed potential approximation is uniformly better for potentials with discontinuities than the $O\left(\hbar^{2}\right)$ approximation.

Smooth QHD simulations of the resonant tunneling diode (RTD) and comparison with experimental current-voltage curves are presented in [8]. Future work will compare the smooth QHD simulations of the RTD with numerical simulations of the coupled quantum Liouville/Poisson equations.

Appendix A. Calculation of $\boldsymbol{G}_{\mathbf{0}}$ and $\boldsymbol{G}_{\mathbf{2}}$. According to (51), (55), and (56), the functions $G_{0}(\xi)$ and $G_{2}(\xi)$ are defined by

$$
G_{m}(\xi)=(2 \pi)^{-\frac{d}{2}} \int_{0}^{1} \int_{R_{p}^{d}} p^{\otimes m} \exp \left\{(\gamma-1)\left(\frac{h^{2}}{8}|\xi|^{2}+\frac{|p|^{2}}{2}\right)\right\} \mu w_{0}\left(p, \frac{h}{2} \xi, \gamma\right) d p d \gamma
$$




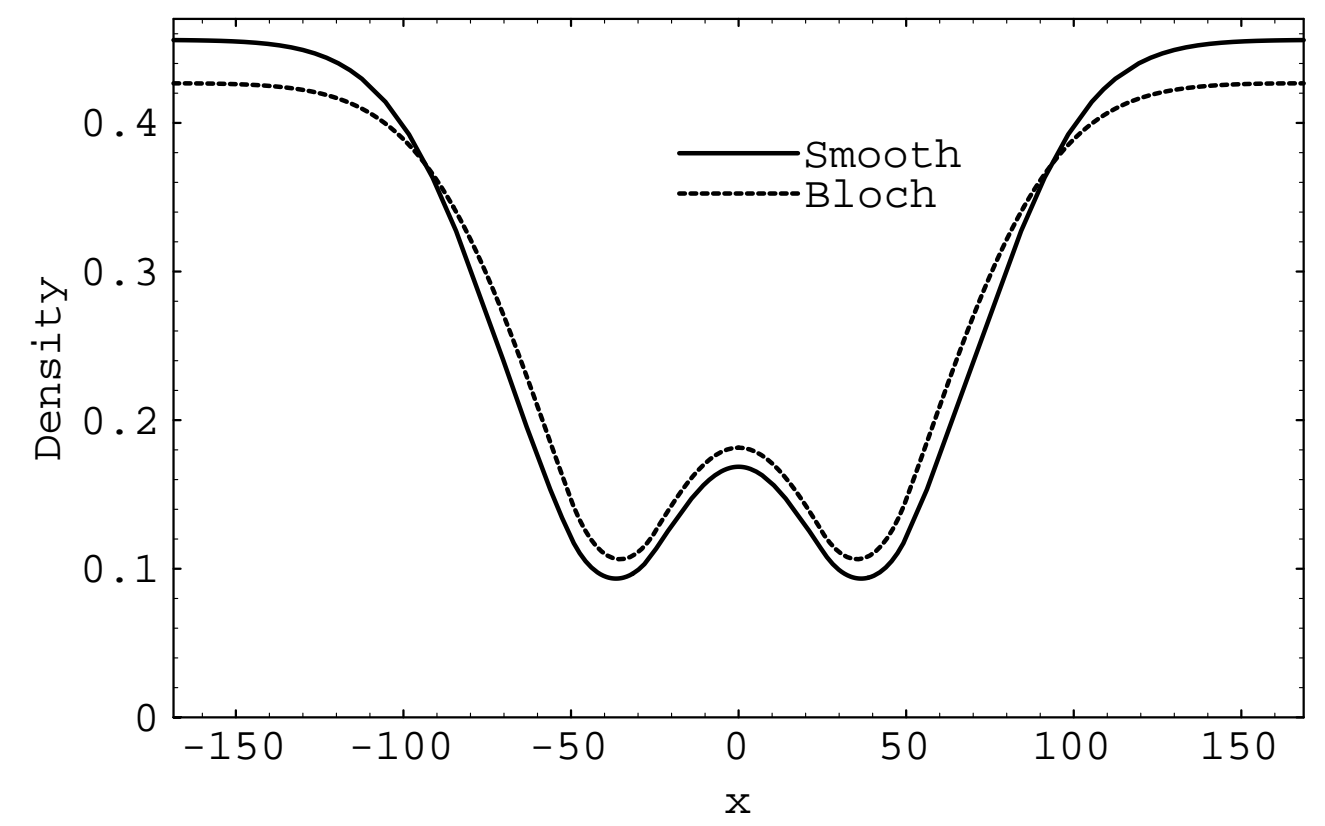

FIG. 5. Electron density in $10^{6} \mathrm{~cm}^{-3}$ for a $0.1 \mathrm{eV}$ double barrier in GaAs at $300 \mathrm{~K}(\varepsilon \approx 2)$.

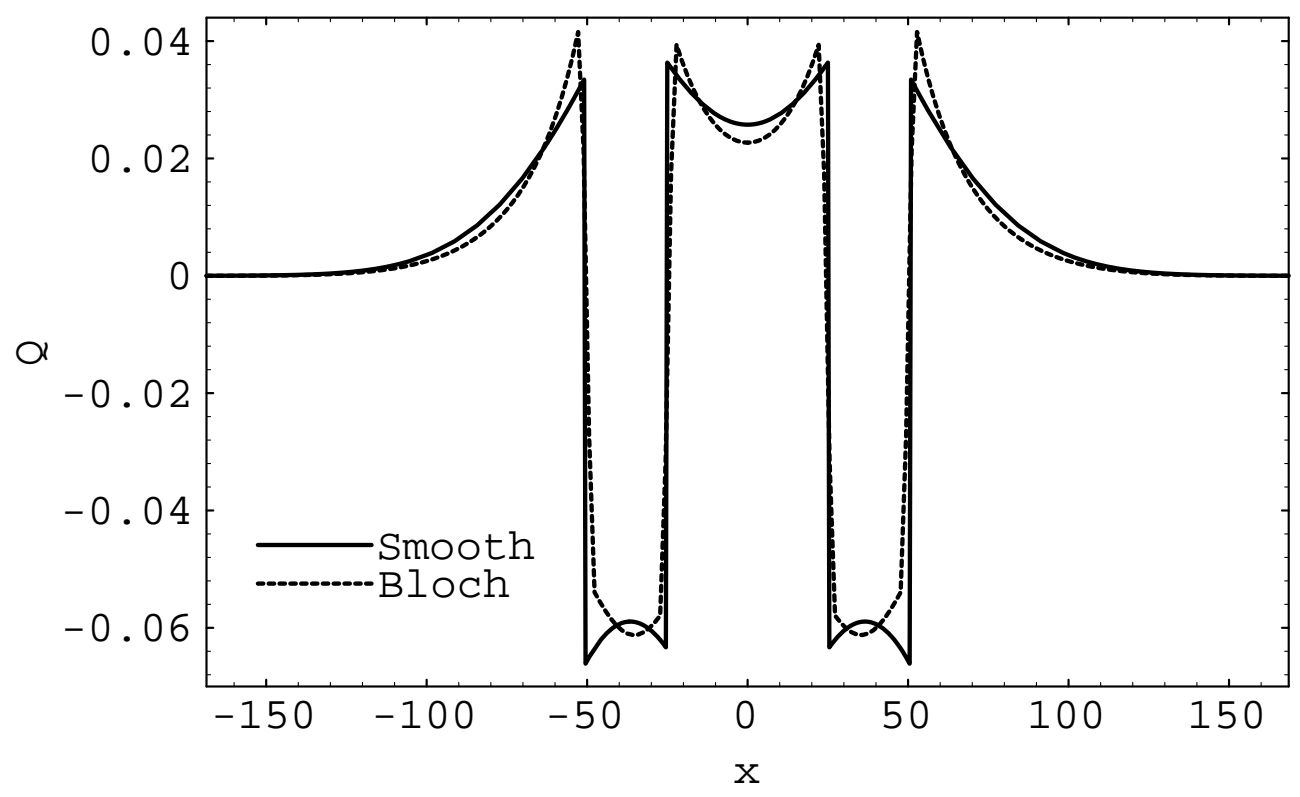

FIG. 6. Quantum correction term in $\mathrm{eV}$ for a $0.1 \mathrm{eV}$ double barrier $(\varepsilon \approx 2)$. 


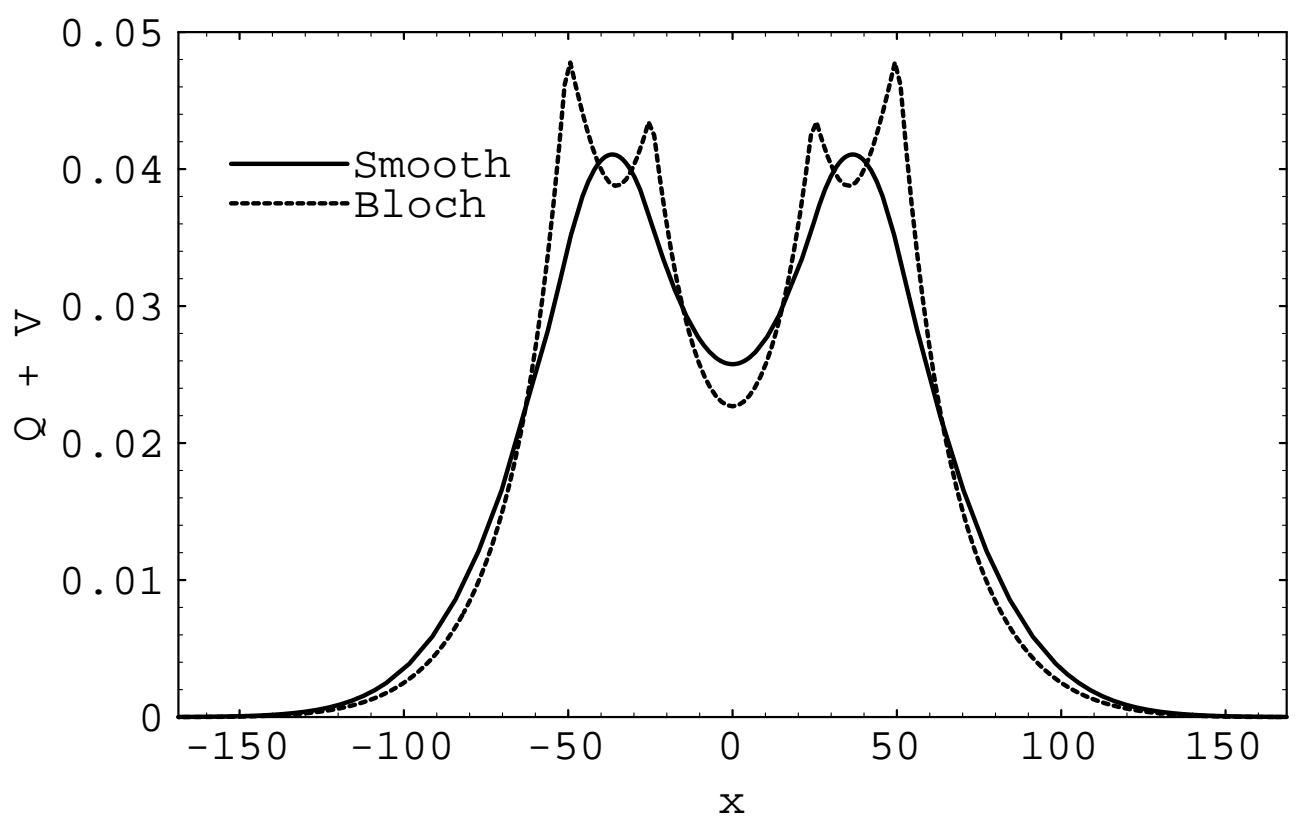

FIG. 7. Smooth effective potential in $\mathrm{eV}$ for a $0.1 \mathrm{eV}$ double barrier $(\varepsilon \approx 2)$.

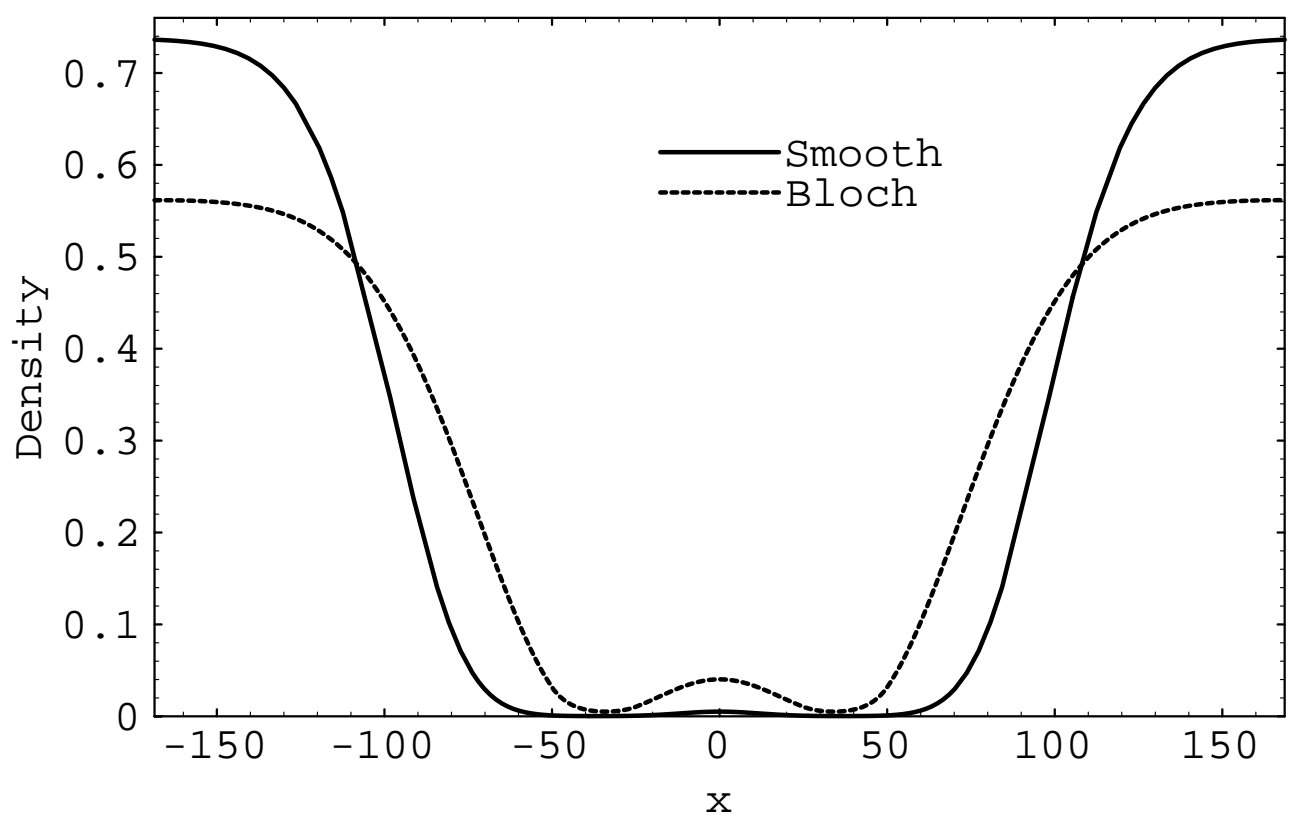

FIG. 8. Electron density in $10^{6} \mathrm{~cm}^{-3}$ for a $0.5 \mathrm{eV}$ double barrier in GaAs at $300 \mathrm{~K}(\varepsilon \approx 10)$. 


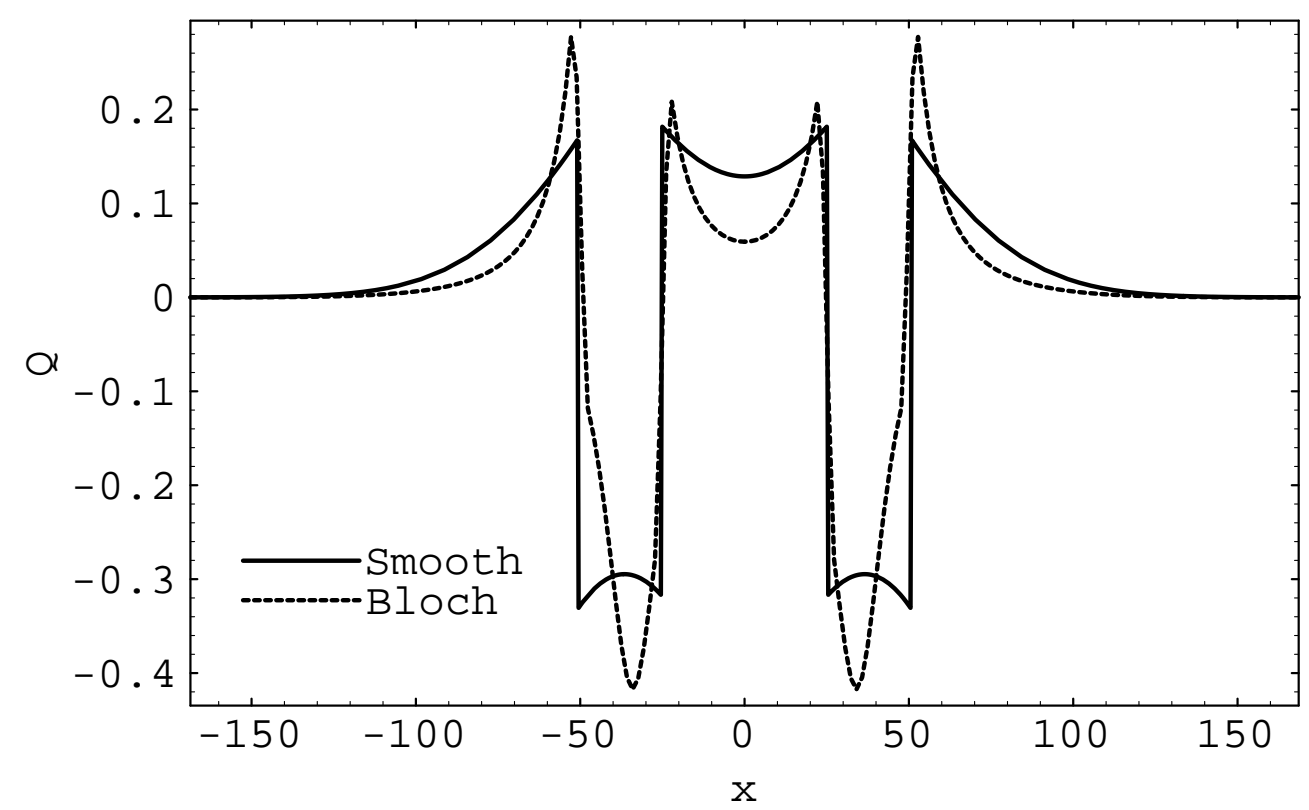

FIG. 9. Quantum correction term in $\mathrm{eV}$ for a $0.5 \mathrm{eV}$ double barrier $(\varepsilon \approx 10)$.

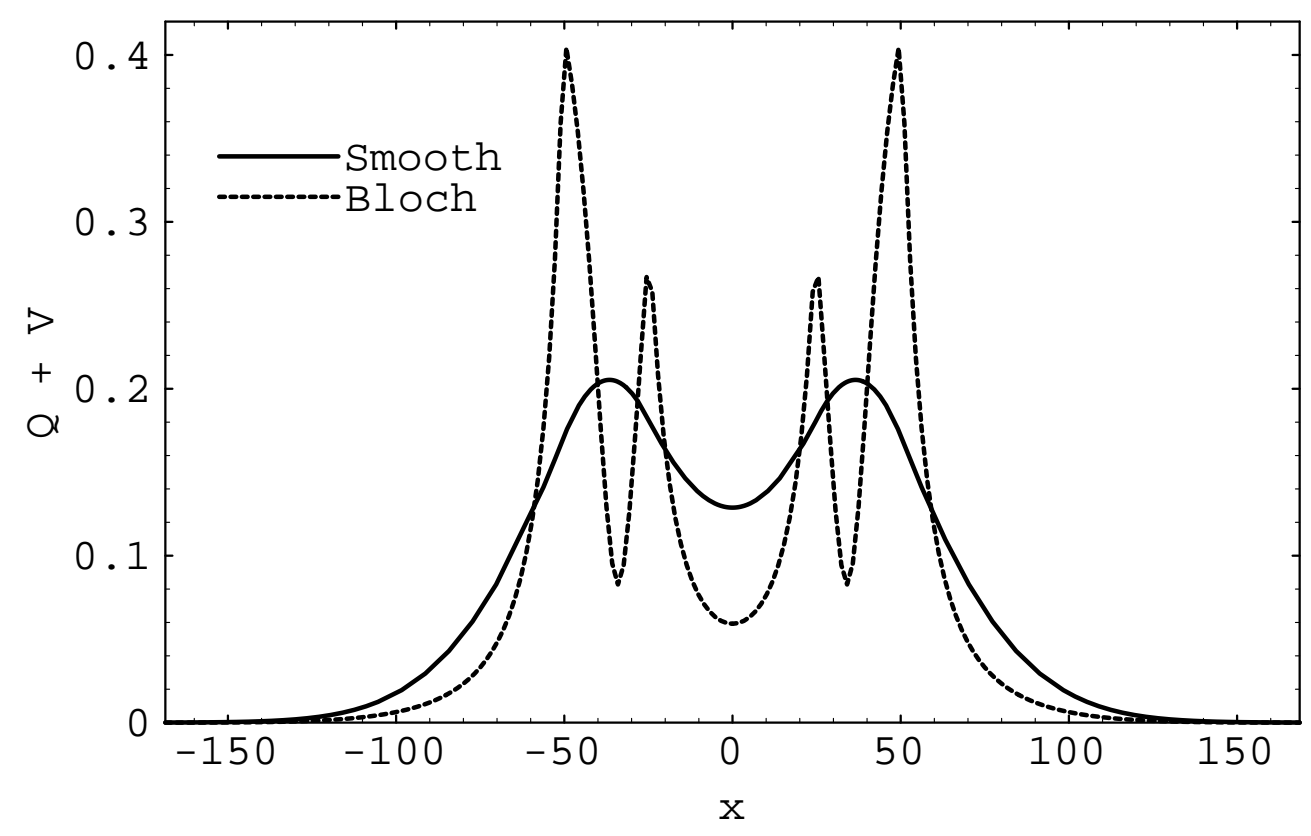

FIG. 10. Smooth effective potential in $\mathrm{eV}$ for a $0.5 \mathrm{eV}$ double barrier $(\varepsilon \approx 10)$. 
with $m=0$, 2. Splitting $G_{m}$ into $G_{m}=\frac{1}{2}\left(G_{m}^{+}+G_{m}^{-}\right)$, we define

$$
G_{m}^{ \pm}(\xi)=(2 \pi)^{-\frac{d}{2}} \int_{0}^{1} \int_{R_{p}^{d}} p^{\otimes m} \exp \left\{(\gamma-1) \frac{h^{2}}{8}|\xi|^{2}\right\} u(p, \gamma) w_{0}\left(p \pm \frac{h}{2} \xi, \gamma\right) d p d \gamma
$$

where

$$
u(p, \gamma)=\exp \left\{\frac{(\gamma-1)}{2}|p|^{2}\right\} .
$$

For $m=0$, integrating with respect to $p$ gives

$$
\begin{gathered}
\int_{R_{p}^{d}} u(p, \gamma) w_{0}\left(p+\frac{h}{2} \xi, \gamma\right) d p=\int_{R_{\eta}^{d}} \tilde{u}(-\eta, \gamma) \tilde{w}_{0}(\eta, \gamma) \exp \left\{i \frac{h}{2} \eta \cdot \xi\right\} d \eta \\
=\int_{R_{\eta}^{d}}[\gamma(1-\gamma)]^{-\frac{d}{2}} \exp \left\{-\frac{1}{2 \gamma(1-\gamma)}|\eta|^{2}+i \frac{h}{2} \eta \cdot \xi\right\} d \eta \\
=(2 \pi)^{\frac{d}{2}} \exp \left\{-\gamma(1-\gamma) \frac{h^{2}}{8}|\xi|^{2}\right\} .
\end{gathered}
$$

Thus

$$
G_{0}^{+}(\xi)=\int_{0}^{1} \exp \left\{\left(\gamma^{2}-1\right) \frac{h^{2}}{8}|\xi|^{2}\right\} d \gamma=\kappa\left(\frac{h}{2}|\xi|\right)
$$

with the function $\kappa$ defined in (59). $G_{0}^{-}$is obtained by replacing $h$ by $-h$, and therefore $G_{0}=G_{0}^{+}=G_{0}^{-}=\kappa(h|\xi| / 2)$.

To compute the matrix $G_{2}^{+}$we have to evaluate the integrals

$$
\begin{gathered}
\int_{R_{p}^{d}} p_{j} p_{k} u(p, \gamma) w_{0}\left(p+\frac{h}{2} \xi, \gamma\right) d p \\
=-\int_{R_{\eta}^{d}}\left[\partial_{j k}^{2} \tilde{u}(-\eta, \gamma)\right] \tilde{w}_{0}(\eta, \gamma) \exp \left\{i \frac{h}{2} \eta \cdot \xi\right\} d \eta \\
=[\gamma(1-\gamma)]^{-\frac{d}{2}} \frac{1}{1-\gamma} \int_{R_{\eta}^{d}}\left[\delta_{j k}-\frac{\eta_{j} \eta_{k}}{1-\gamma}\right] \exp \left\{-\frac{1}{2 \gamma(1-\gamma)}|\eta|^{2}+i \frac{h}{2} \eta \cdot \xi\right\} d \eta \\
=\frac{1}{1-\gamma} \int_{R_{\eta}^{d}}\left(\delta_{j k}-\gamma \eta_{j} \eta_{k}\right) \exp \left\{-\frac{|\eta|^{2}}{2}+i \frac{h}{2} \sqrt{\gamma(1-\gamma)} \eta \cdot \xi\right\} d \eta \\
=(2 \pi)^{\frac{d}{2}} \frac{1}{1-\gamma}\left[\delta_{j k}+\gamma \partial_{z_{j} z_{k}}^{2}\right] \exp \left\{-\frac{|z|^{2}}{2}\right\} \quad \text { at } z=\frac{h}{2} \sqrt{\gamma(1-\gamma)} \xi \\
=(2 \pi)^{\frac{d}{2}}\left[\delta_{j k}+\frac{\gamma}{1-\gamma} z_{j} z_{k}\right] \exp \left\{-\frac{|z|^{2}}{2}\right\} \quad \text { at } z=\frac{h}{2} \sqrt{\gamma(1-\gamma)} \xi .
\end{gathered}
$$


Inserting (122) into (118) gives

$$
G_{2_{j k}}^{+}(\xi)=\int_{0}^{1}\left(\delta_{j k}+\frac{\gamma^{2} h^{2}}{4} \xi_{j} \xi_{k}\right) \exp \left\{\left(\gamma^{2}-1\right) \frac{h^{2}}{8}|\xi|^{2}\right\} d \gamma
$$

and integrating by parts yields

$$
G_{2_{j k}}^{+}(\xi)=|\xi|^{-2}\left[\xi_{j} \xi_{k}+\left(|\xi|^{2} \delta_{j k}-\xi_{j} \xi_{k}\right) \kappa\left(\frac{h}{2}|\xi|\right)\right] .
$$

$G_{2}^{-}$is obtained by replacing $h$ by $-h$, and therefore $G_{2}=G_{2}^{+}=G_{2}^{-}$.

Appendix B. Proof of Theorem 2. To prove Theorem 2 we will need the following two lemmas. First we estimate the principal part of the operator in (71).

Lemma 3. Let the function $u(x, p, \beta)$ satisfy the initial value problem

$$
\partial_{\beta} u=\frac{h^{2}}{8} \Delta_{x} u-\frac{|p|^{2}}{2} u+\left(1-\frac{h^{2}}{8} \Delta_{x}+\frac{|p|^{2}}{2}\right) f, \quad u(x, p, 0)=0,
$$

with $0 \leq \beta \leq 1$. Then $\|u\| \leq$ const $\|f\|$, where const denotes a positive constant independent of $h$ and $\varepsilon$ and where the norm $\|\cdot\|$ is defined in (83).

Proof. The Fourier transform $\tilde{u}$ of the solution of (125) is

$$
\tilde{u}(\xi, p, \beta)=\int_{0}^{\beta} \exp \left\{(\gamma-\beta)\left(\frac{h^{2}}{8}|\xi|^{2}+\frac{|p|^{2}}{2}\right)\right\}\left(1+\frac{h^{2}}{8}|\xi|^{2}+\frac{|p|^{2}}{2}\right) \tilde{f}(\xi, p, \gamma) d \gamma .
$$

Thus

$$
\begin{aligned}
& |\tilde{u}(\xi, p, \beta)| \leq M\left(1+\frac{h^{2}}{8}|\xi|^{2}+\frac{|p|^{2}}{2}\right) \int_{0}^{\beta} \exp \left\{(\gamma-\beta)\left(\frac{h^{2}}{8}|\xi|^{2}+\frac{|p|^{2}}{2}\right)\right\} d \gamma \\
\leq & M\left(1+\frac{h^{2}}{8}|\xi|^{2}+\frac{|p|^{2}}{2}\right)\left[1-\exp \left\{-\beta\left(\frac{h^{2}}{8}|\xi|^{2}+\frac{|p|^{2}}{2}\right)\right\}\right]\left[\frac{h^{2}}{8}|\xi|^{2}+\frac{|p|^{2}}{2}\right]^{-1},
\end{aligned}
$$

where

$$
M=\max _{0 \leq \beta \leq 1}\{|\tilde{f}(\xi, p, \beta)|\}
$$

Since the function $g(z, \beta)=\frac{1+z}{z}\left(1-e^{-\beta z}\right)$ is uniformly bounded for $0 \leq z<\infty$, $0 \leq \beta \leq 1$, we obtain

$$
\max _{0 \leq \beta \leq 1}\{|\tilde{u}(\xi, p, \beta)|\} \leq \text { const } \max _{0 \leq \beta \leq 1}\{|\tilde{f}(\xi, p, \beta)|\} .
$$

Squaring both sides of (129) and integrating with respect to $\xi$ and $p$ proves the lemma.

Next we estimate the inhomogeneous term in (125). 
LEMMA 4. Let the function $f(x, p, \beta)$ be given by

$$
\left(1-\frac{h^{2}}{8} \Delta_{x}+\frac{|p|^{2}}{2}\right) f(x, p, \beta)=\Omega_{\alpha} u
$$

for some function $u$, where the operator $\Omega_{\alpha}$ is defined in (81). Then if the Fourier transform of the potential satisfies $|\tilde{V}(\xi)| \leq \operatorname{const}\left(1+|\xi|^{2}\right)^{-\frac{1}{2}},\|f\| \leq$ const ||$u \|$ for $0 \leq \alpha<3$.

Proof. We split the function $f$ and the operator $\Omega_{\alpha}$ into $f=\frac{1}{2}\left(f_{+}+f_{-}\right), \Omega_{\alpha}=$ $\frac{1}{2}\left(\Omega_{\alpha}^{+}+\Omega_{\alpha}^{-}\right)$, where $f_{ \pm}$and $\Omega_{\alpha}^{ \pm}$are defined by

$$
\begin{gathered}
\Omega_{\alpha}^{ \pm}=\left(1+|p|^{2}\right)^{\frac{\alpha}{2}} V\left(x \pm \frac{h}{2 i} \nabla_{p}\right)\left(1+|p|^{2}\right)^{-\frac{\alpha}{2}}, \\
\left(1-\frac{h^{2}}{8} \Delta_{x}+\frac{|p|^{2}}{2}\right) f_{ \pm}=\Omega_{\alpha}^{ \pm} u .
\end{gathered}
$$

Fourier-transforming the function $f_{+}$with respect to the position variable $x$ yields

$$
\begin{gathered}
\tilde{f}_{+}(\xi, p, \beta)=(2 \pi)^{-\frac{3 d}{2}}\left(1+\frac{h^{2}}{8}|\xi|^{2}+\frac{|p|^{2}}{2}\right)^{-1}\left(1+|p|^{2}\right)^{\frac{\alpha}{2}} \\
\times \int_{R_{x}^{d}} \int_{R_{q}^{d}} \int_{R_{\eta}^{d}} V\left(x+\frac{h}{2} \eta\right)\left(1+|q|^{2}\right)^{-\frac{\alpha}{2}} u(x, q, \beta) e^{i \eta \cdot(p-q)-i \xi \cdot x} d \eta d q d x \\
=(2 \pi)^{-\frac{d}{2}}\left(1+\frac{h^{2}}{8}|\xi|^{2}+\frac{|p|^{2}}{2}\right)^{-1}\left(1+|p|^{2}\right)^{\frac{\alpha}{2}} \\
\times \int_{R_{\omega}^{d}} \tilde{V}(\omega)\left(1+\left|p+\frac{h}{2} \omega\right|^{2}\right)^{-\frac{\alpha}{2}} \tilde{u}\left(\xi-\omega, p+\frac{h}{2} \omega, \beta\right) d \omega .
\end{gathered}
$$

Taking the maximum with respect to $\beta$ and integrating with respect to $p$ yields

$$
\begin{gathered}
\int_{R_{p}^{d}} \max _{0 \leq \beta \leq 1}\left\{\left|\tilde{f}_{+}(\xi, p, \beta)\right|^{2}\right\} d p \\
\leq \max _{\omega, p}\left\{\left(1+\frac{h^{2}}{8}|\xi|^{2}+\frac{|p|^{2}}{2}\right)^{-2}\left(1+|p|^{2}\right)^{\alpha}\left(1+|\omega|^{2}\right)^{-1}\left(1+\left|p+\frac{h}{2} \omega\right|^{2}\right)^{-\alpha}\right\} \\
\times \int_{R_{\omega}^{d}} \int_{R_{p}^{d}} \max _{\beta}\left\{\left|\tilde{u}\left(\xi-\omega, p+\frac{h}{2} \omega, \beta\right)\right|^{2}\right\} d p d \omega
\end{gathered}
$$

or

$$
\int_{R_{p}^{d}} \max _{0 \leq \beta \leq 1}\left\{\left|\tilde{f}_{+}(\xi, p, \beta)\right|^{2}\right\} d p \leq H(\xi)\|u\|^{2},
$$


where

$$
H(\xi)=\max _{\omega, p}\left\{\left(1+\frac{h^{2}}{8}|\xi|^{2}+\frac{|p|^{2}}{2}\right)^{-2}\left(1+|p|^{2}\right)^{\alpha}\left(1+|\omega|^{2}\right)^{-1}\left(1+\left|p+\frac{h}{2} \omega\right|^{2}\right)^{-\alpha}\right\} .
$$

Elementary calculus implies that $\int_{R_{\xi}^{d}} H(\xi) d \xi<\infty$ for $0 \leq \alpha<3$. The lemma follows from integrating (135) with respect to $\xi$ and repeating the same procedure for $f_{-}$.

The proof of Theorem 2 consists of the combination of Lemmas 3 and 4 with the function $u$ in Lemma 4 replaced by $\varepsilon r_{\alpha}+w_{1 \alpha}$.

Appendix C. Proof of Lemma 2. The $x$-Fourier transform of the function $w_{1 \alpha}$ is

$$
\begin{gathered}
\tilde{w}_{1 \alpha}(\xi, p, \beta)=-\left(1+|p|^{2}\right)^{\frac{\alpha}{2}} \tilde{V}(\xi) \\
\times \int_{0}^{\beta} \exp \left\{(\gamma-\beta)\left(\frac{h^{2}}{8}|\xi|^{2}+\frac{|p|^{2}}{2}\right)\right\} \mu w_{0}\left(p, \frac{h}{2} \xi, \gamma\right) d \gamma .
\end{gathered}
$$

We split the function $\tilde{w}_{1 \alpha}$ into $\tilde{w}_{1 \alpha}=\frac{1}{2}\left(\tilde{w}_{1 \alpha}^{+}+\tilde{w}_{1 \alpha}^{-}\right)$, with $w_{1 \alpha}^{ \pm}$defined by

$$
\tilde{w}_{1 \alpha}^{ \pm}=-\left(1+|p|^{2}\right)^{\frac{\alpha}{2}} \tilde{V}(\xi) \int_{0}^{\beta} \exp \left\{(\gamma-\beta)\left(\frac{h^{2}}{8}|\xi|^{2}+\frac{|p|^{2}}{2}\right)-\frac{\gamma}{2}\left|p \pm \frac{h}{2} \xi\right|^{2}\right\} d \gamma
$$

and obtain for $\tilde{w}_{1 \alpha}^{+}$

$$
\begin{aligned}
& \tilde{w}_{1 \alpha}^{+}=-2\left(1+|p|^{2}\right)^{\frac{\alpha}{2}} \tilde{V}(\xi)\left[\left|p+\frac{h}{2} \xi\right|^{2}-\left(|p|^{2}+\frac{h^{2}}{4}|\xi|^{2}\right)\right]^{-1} \\
& \times\left[\exp \left\{-\frac{\beta}{2}\left|p+\frac{h}{2} \xi\right|^{2}\right\}-\exp \left\{-\frac{\beta}{2}\left(|p|^{2}+\frac{h^{2}}{4}|\xi|^{2}\right)\right\}\right]
\end{aligned}
$$

The function $g(\beta)=\left(e^{-\beta b}-e^{-\beta a}\right) /(a-b)$ satisfies

$$
0 \leq g(\beta) \leq \exp \left\{\frac{b \ln (b)-a \ln (a)}{a-b}\right\} \leq \text { const } \frac{1}{1+a}
$$

for $0 \leq b \leq 2 a$. Thus

$$
\left|\tilde{w}_{1 \alpha}^{+}(\xi, p, \beta)\right| \leq \operatorname{const}\left(1+|p|^{2}\right)^{\frac{\alpha}{2}}|\tilde{V}(\xi)|\left[1+|p|^{2}+\frac{h^{2}}{4}|\xi|^{2}\right]^{-1}
$$

and

$$
\left\|w_{1 \alpha}^{+}\right\|^{2} \leq \int_{R_{\xi}^{d}} \int_{R_{p}^{d}}\left(1+|p|^{2}\right)^{\frac{\alpha}{2}}|\tilde{V}(\xi)|^{2}\left[1+|p|^{2}+\frac{h^{2}}{4}|\xi|^{2}\right]^{-2} d p d \xi .
$$

The integral on the right-hand side of (142) is convergent for $0 \leq \alpha<\frac{3}{2}$. Repeating the same argument for $w_{1 \alpha}^{-}$proves the lemma. 


\section{REFERENCES}

[1] A. ARnold, The relaxation-time Wigner equation, in Mathematical Problems in Semiconductor Physics, Pitman Res. Notes Math. Ser. 340, Rome, 1995, pp. 105-117.

[2] A. ARnold And F. Nier, The two-dimensional Wigner-Poisson problem for an electron gas in the charge neutral case, Math. Methods Appl. Sci., 14 (1991), pp. 595-613.

[3] D. K. Ferry and J.-R. Zhou, Form of the quantum potential for use in hydrodynamic equations for semiconductor device modeling, Phys. Rev. B, 48 (1993), pp. 7944-7950.

[4] R. P. Feynman and H. Kleinert, Effective classical partition functions, Phys. Rev. A, 34 (1986), pp. 5080-5084.

[5] C. L. GaRdner, The quantum hydrodynamic model for semiconductor devices, SIAM J. Appl. Math., 54 (1994), pp. 409-427.

[6] C. L. Gardner, A. Niemic, and C. Ringhofer, Numerical methods for the Bloch equation, to appear.

[7] C. L. Gardner and C. Ringhofer, Smooth quantum potential for the hydrodynamic model, Phys. Rev. E, 53 (1996), pp. 157-167.

[8] C. L. Gardner and C. RInghofer, Smooth QHD simulation of the resonant tunneling diode, VLSI Design, (1998), to appear.

[9] H. L. Grubin, T. R. Govindan, J. P. Kreskovsky, and M. A. Stroscio, Transport via the Liouville equation and moments of quantum distribution functions, Solid-State Electronics, 36 (1993), pp. 1697-1709.

[10] T. Kerkhoven, M. W. Raschke, and U. Ravaioli, Self-consistent simulation of corrugated layered structures, Superlattices and Microstructures, 12 (1992), pp. 505-508.

[11] N. C. Kluksdahl, A. M. Kriman, D. K. Ferry, and C. Ringhofer, Self-consistent study of the resonant-tunneling diode, Phys. Rev. B, 39 (1989), pp. 7720-7735.

[12] N. J. Mauser And C. Ringhofer, Quantum steady states via self-consistent Bloch-type equations, in Proc. International Workshop on Computational Electronics, University of Illinois, Urbana-Champaign, 1992, pp. 273-276.

[13] N. J. Mauser, C. Ringhofer, and G. Ulich, Numerical methods for Bloch-Poisson type equations, in Proc. International Workshop on Computational Electronics, University of Leeds, UK, 1993, pp. 291-295.

[14] E. Wigner, On the quantum correction for thermodynamic equilibrium, Phys. Rev. 40 (1932), pp. 749-759. 